\title{
HYPERBOLIC DISTANCE VERSUS QUASIHYPERBOLIC DISTANCE IN PLANE DOMAINS
}

\author{
DAVID A. HERRON AND JEFF LINDQUIST
}

Dedicated to David Minda, for decades of interesting discussions.

\begin{abstract}
We examine Euclidean plane domains with their hyperbolic or quasihyperbolic distance. We prove that the associated metric spaces are quasisymmetrically equivalent if and only if they are bi-Lipschitz equivalent. On the other hand, for Gromov hyperbolic domains, the two corresponding Gromov boundaries are always quasisymmetrically equivalent. Surprisingly, for any finitely connected hyperbolic domain, these two metric spaces are always quasiisometrically equivalent. We construct examples where the spaces are not quasiisometrically equivalent.
\end{abstract}

\section{INTRODUCTION}

Throughout this section $\Omega$ denotes a hyperbolic plane domain: $\Omega \subset \mathbb{C}$ is open and connected and $\mathbb{C} \backslash \Omega$ contains at least two points. Each such $\Omega$ carries a unique maximal constant curvature -1 conformal metric $\lambda d s=\lambda_{\Omega} d s$ usually referred to as the Poincaré hyperbolic metric on $\Omega$. The length distance $h=h_{\Omega}$ induced by $\lambda d s$ is called hyperbolic distance in $\Omega$. There is also a quasihyperbolic metric $\delta^{-1} d s=\delta_{\Omega}^{-1} d s$ on $\Omega$, whose length distance $k=k_{\Omega}$ is called quasihyperbolic distance in $\Omega$; here $\delta(z)=\delta_{\Omega}(z):=\operatorname{dist}(z, \partial \Omega)$ is the Euclidean distance from $z$ to the boundary of $\Omega$. See $₫ 2$.C for more details.

This work continues that begun in BH20, Her21a, Her21b where we elucidate the geometric similarities and metric differences between the metric spaces $(\Omega, h)$ and $(\Omega, k)$. Our first result, Theorem $\mathrm{A}$ below, characterizes when the metric spaces $(\Omega, h)$ and $(\Omega, k)$ are quasisymmetrically equivalent. Theorem $\mathrm{B}$ reveals that, when these spaces are Gromov hyperbolic, their Gromov boundaries are always quasisymmetrically equivalent.

To set the stage, we begin with some preliminary observations. A straightforward, albeit non-trivial, argument reveals that the metric spaces $(\Omega, h)$ and $(\Omega, k)$ are isometric if and only if $\Omega$ is an open half-plane and the isometry is the restriction of a Möbius transformation. Furthermore, these metric spaces are bi-Lipschitz equivalent if and only if the identity map is bi-Lipschitz; see \$2.C.3.

It is well-known that the identity $\operatorname{map}(\Omega, k) \stackrel{\text { id }}{\rightarrow}(\Omega, h)$ enjoys the following properties:

- The map id is a 2-Lipschitz 1-quasiconformal homeomorphism.

- For any simply connected $\Omega$, id is 2-bi-Lipschitz 1

Received by the editors October 7, 2020, and, in revised form, March 23, 2021.

2020 Mathematics Subject Classification. Primary 30F45, 30L99; Secondary 51F99, 30 C62.

Key words and phrases. Hyperbolic metric, quasihyperbolic metric, quasisymmetry, quasiisometry.

${ }^{1}$ That $\mathrm{id}^{-1}$ is 2-Lipschitz is a consequence of Koebe's One Quarter Theorem. 
- In general, id is bi-Lipschitz if and only if $\hat{\mathbb{C}} \backslash \Omega$ is uniformly perfect 2

The last item above is due to Beardon and Pommerenke; see BP78] and $\$ 2$. C.3.

Our interest is in general (non-simply connected) hyperbolic plane domains $\Omega$ where there may be no simple metric control on $\mathrm{id}^{-1}$. For example, given any sequences $\left(h_{n}\right)_{1}^{\infty}$ and $\left(k_{n}\right)_{1}^{\infty}$ of positive numbers with say $1 \geq h_{n} \rightarrow 0$ and $2 \leq$ $k_{n} \rightarrow \infty$, there are sequences $\left(a_{n}\right)_{1}^{\infty},\left(b_{n}\right)_{1}^{\infty}$ of points in the punctured unit disk $\mathbb{D}_{\star}:=\mathbb{D} \backslash\{0\}$ with hyperbolic and quasihyperbolic distances $h_{\star}\left(a_{n}, b_{n}\right)=h_{n}$ and $k_{\star}\left(a_{n}, b_{n}\right)=k_{n}$. See [BH20, Ex. 2.7].

The following striking rigidity theorem contains our first main result. (See $2 . \mathrm{A}$ for mapping definitions.) This says that the metric spaces $(\Omega, k)$ and $(\Omega, h)$ are either "quite similar" (i.e., bi-Lipschitz equivalent) or "quite different" (i.e., not quasisymmetrically equivalent).

Theorem A. For any hyperbolic plane domain $\Omega$, the following are quantitatively equivalent.

(A.1) The metric spaces $(\Omega, k)$ and $(\Omega, h)$ are quasisymmetrically equivalent.

(A.2) The metric spaces $(\Omega, k)$ and $(\Omega, h)$ are bi-Lipschitz equivalent.

(A.3) The identity map $(\Omega, k) \rightarrow(\Omega, h)$ is bi-Lipschitz.

(A.4) $\hat{\mathbb{C}} \backslash \Omega$ is uniformly perfect.

Again, Beardon and Pommerenke [BP78] established the equivalence of $[\mathrm{A}] 3$ ) and $(\mathrm{A}, 4)$.

Recently, the first author and Buckley [BH20, Theorem B] demonstrated that $(\Omega, k)$ and $(\Omega, h)$ are simultaneously Gromov hyperbolic or not, and we call $\Omega$ Gromov hyperbolic in the former case. Our next result stands in stark contrast to Theorem $\mathrm{A}$. Even when $(\Omega, k)$ and $(\Omega, h)$ are not quasisymmetrically equivalent, the large scale geometry is the same in both spaces - at least when they are Gromov hyperbolic. This further enhances $[\mathrm{BH} 20$, Theorem $\mathrm{A}]$ where we proved that these metric spaces have the same quasi-geodesic curves.

Theorem B. For any Gromov hyperbolic plane domain $\Omega$, the canonical conformal gauges on the Gromov boundaries $\partial_{G}(\Omega, k)$ and $\partial_{G}(\Omega, h)$ are naturally quasisymmetrically equivalent.

If $(\Omega, h)$ and $(\Omega, k)$ are quasiisometrically 3 equivalent, the above Gromov boundary equivalence is known and given via a power quasisymmetry; see BS00, Theorem 6.5(2)]. Whereas our proof of Theorem $\mathrm{A}$ is surprisingly simple, the proof of Theorem B employs significant machinery as explained in the first paragraph of $3 . \mathrm{B}$

Again, when $\hat{\mathbb{C}} \backslash \Omega$ is uniformly perfect, $(\Omega, k)$ and $(\Omega, h)$ are bi-Lipschitz equivalent. A natural conjecture is that this uniform perfectness might be a necessary condition for $(\Omega, k)$ and $(\Omega, h)$ to be quasiisometrically equivalent. However, our next result reveals that this is not the case.

Theorem C. For any finitely connected hyperbolic plane domain $\Omega$, the metric spaces $(\Omega, k)$ and $(\Omega, h)$ are quasiisometrically equivalent.

The above is just an easy to state special case of our more general Theorem 3.9 which provides a large class of plane domains whose hyperbolizations and quasihyperbolizations are quasiisometrically equivalent. This raises the natural question

\footnotetext{
${ }^{2}$ The bi-Lipschitz and uniformly perfectness constants depend only on each other.

${ }^{3}$ Our quasiisometries are sometimes called rough bi-Lipschitz maps.
} 
of whether the conclusion of Theorem $\mathrm{C}$ could be true in general, and we answer this below. Note that a quasiisometry can have an arbitrarily large additive rough constant and this obstacle must be overcome.

Theorem D. There are uniform (hence Gromov) hyperbolic plane domains $\Omega$ with the property that any quasisymmetric equivalence between $\partial_{G}(\Omega, k)$ and $\partial_{G}(\Omega, h)$, e.g., that given by Theorem $\mathrm{B}$, is not via a power quasisymmetry. In particular, $(\Omega, k)$ and $(\Omega, h)$ are not quasiisometrically equivalent.

Domains that satisfy Theorem $\mathrm{D}$ include any $\Omega:=\mathbb{C} \backslash\left\{a_{n}\right\}_{0}^{\infty}$, where, $a_{0}:=0$, and $\left(a_{n}\right)_{1}^{\infty}$ is a strictly decreasing sequence in $(-\infty, 0)$ with $a_{n+1} / a_{n} \rightarrow+\infty$ as $n \rightarrow+\infty$

Section 2 contains the usual definitions and terminology; especially, see 2.C.1 and $\$ 2 . \mathrm{C.2}$ for details about the hyperbolic and quasihyperbolic metrics. We prove Theorems A, B, C, D in $\S ₫ 3 . \mathrm{A}, 3 . \mathrm{B}$, 3.C, 3.D respectively.

\section{Preliminaries}

We work in the Euclidean plane, and on the Riemann sphere, which we identify, respectively, with the complex number field $\mathbb{C}$ and its one-point extension $\hat{\mathbb{C}}:=$ $\mathbb{C} \cup\{\infty\}$. Everywhere $\Omega$ is a domain 4 (i.e., an open connected set) and $\partial \Omega$ and $\hat{\partial} \Omega$ denote the boundary of $\Omega$ with respect to the plane and sphere (respectively). Always, $\Omega$ is a hyperbolic domain, i.e., $\hat{\mathbb{C}} \backslash \Omega$ contains at least three points.

We write $\hat{A}$ and $\hat{\partial} A$ for the closure and boundary of a set $A$ in $\hat{\mathbb{C}}$ whereas $\bar{A}$ and $\partial A$ are the Euclidean closure and boundry of $A$.

We write $C=C(D, \ldots)$ to indicate a constant $C$ that depends only on the data $D, \ldots$ In some cases we write $K_{1} \lesssim K_{2}$ to indicate that $K_{1} \leq C K_{2}$ for some computable constant $C$ that depends only on the relevant data, and $K_{1} \simeq K_{2}$ means $K_{1} \lesssim K_{2} \lesssim K_{1}$.

The Euclidean line segment joining two points $a, b$ is $[a, b]$, and $(a, b)=[a, b] \backslash$ $\{a, b\}$. The open and closed Euclidean disks, and the circle, centered at the point $a \in \mathbb{C}$ and of radius $r>0$, are denoted by $\mathrm{D}(a ; r)$ and $\mathrm{D}[a ; r]$ and $\mathrm{S}^{1}(a ; r)$ respectively, and $\mathbb{D}:=\mathrm{D}(0 ; 1)$ is the unit disk. We also define

$$
\mathbb{C}_{\star}:=\mathbb{C} \backslash\{0\}, \quad \mathbb{C}_{a b}:=\mathbb{C} \backslash\{a, b\}, \quad \mathbb{D}_{\star}:=\mathbb{D} \backslash\{0\}, \quad \mathbb{D}^{\star}:=\mathbb{C} \backslash \overline{\mathbb{D}} ;
$$

the definition of $\mathbb{C}_{a b}$ is for distinct points $a, b$ in $\mathbb{C}$.

The quantity $\delta(z)=\delta_{\Omega}(z):=\operatorname{dist}(z, \partial \Omega)$ is the Euclidean distance from $z \in \mathbb{C}$ to the boundary of $\Omega$, and $1 / \delta$ is the scaling factor (aka, metric-density) for the so-called quasihyperbolic metric $\delta^{-1} d s$ on $\Omega \subset \mathbb{C}$; see $\$ 2 . C .1$. We use the notation

$$
\mathrm{D}(z)=\mathrm{D}_{\Omega}(z):=\mathrm{D}(z ; \delta(z))=\mathrm{D}\left(z ; \delta_{\Omega}(z)\right)
$$

for the maximal Euclidean disk in $\Omega$ centered at a point $z \in \Omega$, and then

$$
\mathrm{B}(z)=\mathrm{B}_{\Omega}(z):=\partial \mathrm{D}(z) \cap \partial \Omega=\mathrm{S}^{1}(z ; \delta(z)) \cap \partial \Omega
$$

is the set of all nearest boundary points for $z$.

\footnotetext{
${ }^{4}$ Our interest is in non-simply connected domains $\Omega$ primarily with $\hat{\mathbb{C}} \backslash \Omega$ non-uniformly perfect.
} 
The chordal and spherical distances on $\hat{\mathbb{C}}$ are $\chi$ and $\sigma$, respectively. Thus

$$
\chi(z, w):= \begin{cases}\frac{2|z-w|}{\sqrt{1+|z|^{2}} \sqrt{1+|w|^{2}}} & \text { if } z, w \in \mathbb{C} \\ \frac{2}{\sqrt{1+|z|^{2}}} & \text { if } z \in \mathbb{C}, w=\infty\end{cases}
$$

and $\sigma$ is the length distance associated with $\chi 57 \quad \chi=2 \sin (\sigma / 2)$, and $\chi \leq \sigma \leq$ $(\pi / 2) \chi$. Calculations are easier with $\chi$, but $\sigma$ is a geodesic distance whereas $\chi$ is not geodesic.

Each of the metric spaces $(\Omega,|\cdot|),(\Omega, \chi),(\Omega, \sigma)$ has an associated length distance, although the latter two are equal. We write $l=l_{\Omega}$ for the intrinsic (aka, inner) Euclidean length distance and $l_{\sigma}=l_{\Omega_{\sigma}}$ for the intrinsic chordal length distance (which equals the intrinsic spherical length distance), and then $(\Omega, l)$ and $\left(\Omega, l_{\sigma}\right)$ are the corresponding length spaces. See for example Her10.

It is convenient to let $\chi(z)$ and $\sigma(z)$ denote the chordal and spherical distances from $z$ to $\hat{\partial} \Omega$. Again, $\chi(z) \leq \sigma(z) \leq(\pi / 2) \chi(z)$, and we note that

$$
\sigma(z):=\operatorname{dist}_{\sigma}(z, \hat{\partial} \Omega)=\operatorname{dist}_{l_{\sigma}}\left(z, \partial\left(\Omega, l_{\sigma}\right)\right)
$$

where $\partial\left(\Omega, l_{\sigma}\right)$ is the metric boundary of $\left(\Omega, l_{\sigma}\right)$.

2.A. Maps, paths, and geodesics. An embedding $X \stackrel{f}{\rightarrow} Y$ between two metric spaces is a quasisymmetry if there is a homeomorphism $\eta:[0, \infty) \rightarrow[0, \infty)$ (called a distortion function) such that for all triples $x, y, z \in X$,

$$
|x-y| \leq t|x-z| \Longrightarrow|f x-f y| \leq \eta(t)|f x-f z| ;
$$

when this holds, we say that $f$ is $\eta$-QS. These mappings were studied by Tukia and Väisälä in TV80; see also Hei01.

The bi-Lipschitz maps form an important subclass of the quasisymmetric maps; $X \stackrel{f}{\rightarrow} Y$ is bi-Lipschitz if and only if there is a constant $L$ such that for all $x, y \in X$,

$$
L^{-1}|x-y| \leq|f x-f y| \leq L|x-y|,
$$

and when this holds we say that $f$ is $L$-bi-Lipschitz; such an $f$ is $\eta$-QS with $\eta(t):=$ Lt.

More generally, a map $X \stackrel{f}{\rightarrow} Y$ is an $(L, C)$-quasiisometry if $L \geq 1, C \geq 0$ and for all $x, y \in X$,

$$
L^{-1}|x-y|-C \leq|f x-f y| \leq L|x-y|+C .
$$

These are often called rough bi-Lipschitz maps, and there seems to be no universal agreement regarding this terminology; some authors use the adjective quasiisometry to mean what we have called bi-Lipschitz, and then a rough quasiisometry satisfies our definition of quasiisometry. A $(1,0)$-quasiisometry is simply an isometry (onto its range), and a $(1, C)$-quasiisometry is called a $C$-rough isometry.

Two metric spaces $X, Y$ are isometrically equivalent (or BL, QS, $Q C$ equivalent, respectively) if and only if there is a bijection $X \rightarrow Y$ that is an isometry (or BL, QS, or QC).

Also, $X, Y$ are quasiisometrically equivalent if and only if there is a quasiisometry $f: X \rightarrow Y$ with the property that $f(X)$ is cobounded in $Y$ (i.e., the Hausdorff

\footnotetext{
${ }^{5}$ Identifying $\hat{\mathbb{C}}$ with the unit sphere in $\mathbb{R}^{3}$ we see that $\sigma(u, v)$ is the angle between $u, v$.
} 
distance between $f(X)$ and $Y$ is finite). More precisely: $X, Y$ are $(L, C)$-QI equivalent if there is an $(L, C)$-quasiisometry $f: X \rightarrow Y$ and for each $y \in Y$ there is an $x \in X$ with $|y-f(x)| \leq C$. An alternative way to describe this is to say that there are quasiisometries in both directions that are rough inverses of each other.

2.1. Example. Suppose $X \subset Y$ and for each $y \in Y$ there is an $x_{y} \in X$ with $\left|x_{y}-y\right| \leq C$. The maps

$$
X \stackrel{\text { id }}{\hookrightarrow} Y \stackrel{f}{\rightarrow} X, \quad \text { where } \quad f(y):=\left\{\begin{array}{ll}
y & \text { if } y \in X \\
x_{y} & \text { if } y \notin X
\end{array},\right.
$$

are both rough isometric equivalences: the "identity" inclusion is a $(1, C)$-QI equivalence and $f$ is a $(1,2 C)$-QI equivalence.

Our metric spaces will always be the domain $\Omega$, either in $\mathbb{C}$ or in $\hat{\mathbb{C}}$, with either Euclidean distance, chordal distance, spherical distance, an associated length distance, an associated quasihyperbolic distance, or an associated hyperbolic distance.

A path in $X$ is a continuous map $\mathbb{R} \supset I \stackrel{\gamma}{\rightarrow} X$ where $I=I_{\gamma}$ is an interval (called the parameter interval for $\gamma$ ) that may be closed or open or neither and finite or infinite. The trajectory of such a path $\gamma$ is $|\gamma|:=\gamma(I)$ which we call a curve. When $I$ is closed and $I \neq \mathbb{R}, \partial \gamma:=\gamma(\partial I)$ denotes the set of endpoints of $\gamma$ which consists of one or two points depending on whether or not $I$ is compact. For example, if $I_{\gamma}=[0,1] \subset \mathbb{R}$, then $\partial \gamma=\{\gamma(0), \gamma(1)\}$

We call $\gamma$ a compact path if its parameter interval $I$ is compact (which we often assume to be $[0,1])$. We call $\gamma$ a rectifiable path if its length $\ell(\gamma)$ is finite, and then we may assume that $\gamma$ is parameterized with respect to arclength in which case the parameter interval for $\gamma$ is $[0, \ell(\gamma)]$. We note that arclength parameterizations are a priori 1-Lipschitz continuous.

When $\partial \gamma=\{a, b\}$, we write $\gamma: a \curvearrowright b($ in $\Omega)$ to indicate that $\gamma$ is a path (in $\Omega)$ with initial point $a$ and terminal point $b$; this notation implies an orientation: $a$ precedes $b$ on $\gamma$.

When $\alpha: a \curvearrowright b$ and $\beta: b \curvearrowright c$ are paths that join $a$ to $b$ and $b$ to $c$ respectively, $\alpha \star \beta$ denotes the concatenation of $\alpha$ and $\beta$; so $\alpha \star \beta: a \curvearrowright c$. Of course, $|\alpha \star \beta|=$ $|\alpha| \cup|\beta|$. Also, the reverse of $\gamma$ is the path $\gamma^{-1}$ defined by $\gamma^{-1}(t):=\gamma(1-t)$ (when $\left.I_{\gamma}=[0,1]\right)$ and going from $\gamma(1)$ to $\gamma(0)$. Of course, $\left|\gamma^{-1}\right|=|\gamma|$.

An $\operatorname{arc} \alpha$ is an injective compact path. Every arc is taken to be ordered from its initial point to its terminal point. Given points $a, b \in|\alpha|$, there are unique $u, v \in I$ with $\alpha(u)=a, \alpha(v)=b$ and we write $\alpha[a, b]:=\left.\alpha\right|_{[u, v]}$. Every compact path contains an arc with the same endpoints; see [Väi94.

A path $I \stackrel{\gamma}{\rightarrow} X$ into a metric space $X$ is a geodesic if $\gamma$ is an isometry (for all $s, t \in I,|\gamma(s)-\gamma(t)|=|s-t|)$ and a $K$-quasi-geodesic if $\gamma$ is $K$-bi-Lipschitz 6

$$
\text { for all } s, t \in I, \quad K^{-1}|s-t| \leq|\gamma(s)-\gamma(t)| \leq K|s-t| .
$$

A characteristic property of geodesics is that the length of each subpath equals the distance between its endpoints. There is a corresponding description for quasigeodesics: $I \stackrel{\gamma}{\rightarrow} X$ is an $L$-chordarc path if it is rectifiable and

$$
\text { for all } s, t \in I, \quad \ell\left(\left.\gamma\right|_{[s, t]}\right) \leq L|\gamma(s)-\gamma(t)| \text {. }
$$

\footnotetext{
${ }^{6}$ One can also consider rough-quasi-geodesics where $\gamma$ is a quasiisometry; we do not do so.
} 
If we ignore parameterizations, then the class of all quasi-geodesics (in some metric space) is exactly the same as the class of all chordarc paths. More precisely, a $K$ quasi-geodesic is a $K^{2}$-chordarc path, and if we parameterize an $L$-chordarc path with respect to arclength, then we get an $L$-quasi-geodesic.

In this paper we study the metric spaces $(\Omega, h)$ or $(\Omega, k)$ where $\Omega$ is a hyperbolic plane domain and $h$ and $k$ are the hyperbolic and quasihyperbolic distances in $\Omega$. The geodesics and quasi-geodesics in $(\Omega, h)$ are called hyperbolic geodesics and hyperbolic quasi-geodesics, and similarly in $(\Omega, k)$ we attach the adjective quasihyperbolic.

2.B. Annuli and uniformly perfect sets. Given $c \in \mathbb{C}$ and $0<r<R<+\infty$, $A:=\{z \in \mathbb{C}|r<| z-c \mid<R\}$ is an Euclidean annulus with center $c(A):=c$ and conformal modulus $\bmod (A):=\log (R / r)$; if $r=0$ or $R=\infty, A$ is a degenerate annulus and $\bmod (A):=+\infty$. We call $\mathrm{S}^{1}(A):=\mathrm{S}^{1}(c ; \sqrt{r R})$ the conformal center circle of $A ; A$ is symmetric about this circle 7 The inner and outer boundary circles of $A$ are, respectively,

$$
\partial_{\text {in }} A:=\mathrm{S}^{1}(c ; r) \quad \text { and } \quad \partial_{\text {out }} A:=\mathrm{S}^{1}(c ; R) .
$$

A point $z$ is inside (outside) $A$ if and only if $z \in A_{\text {in }}:=\mathrm{B}[c ; r]\left(z \in A_{\text {out }}:=\right.$ $\mathbb{C} \backslash \mathrm{B}(c ; R)$ ); that is, $z$ is inside (or outside) $A$ if and only if $z$ is inside $\partial_{\text {in }} A$ (or outside $\left.\partial_{\text {out }} A\right)$.

It is convenient to introduce the notation

$$
A=\mathrm{A}(c ; d, m):=\left\{z \in \mathbb{C}: d e^{-m}<|z-c|<d e^{m}\right\} \quad \text { and } \quad \mathrm{A}[c ; d, m]:=\overline{\mathrm{A}(c ; d, m)} \text {. }
$$

Then $\mathrm{S}^{1}(A)=\mathrm{S}^{1}(c ; d), \partial_{\text {in }} A=\mathrm{S}^{1}\left(c ; d e^{-m}\right)$, and $\partial_{\text {out }} A=\mathrm{S}^{1}\left(c ; d e^{m}\right)$; here $c=c(A)$, $d>0, m>0$.

An annulus $A^{\prime}$ is a subannulus of $A$, denoted by $A^{\prime}$ a $A$, provided

$$
A^{\prime} \subset A \text { and } A_{\text {in }} \subset A_{\text {in }}^{\prime} \text { and } A_{\text {out }} \subset A_{\text {out }}^{\prime} .
$$

Two annuli are concentric if they have a common center, and $A^{\prime}$ is a concentric subannulus of $A$, denoted by $A^{\prime} \subset \mathrm{c} A$, provided $c\left(A^{\prime}\right)=c(A)$ and $A^{\prime}$ is a subannulus of $A$.

An annulus $A$ separates $E$ if $A \subset \hat{\mathbb{C}} \backslash E$ and both components of $\hat{\mathbb{C}} \backslash A$ contains points of $E$; thus when $A$ separates $\{a, b\}$, one of $a$ or $b$ lies inside $A$ and the other lies outside $A$, and if $A$ does not meet nor separate $\{a, b\}$, then $a$ and $b$ are on the same side of $A$. Evidently, if $A^{\prime}$ is a subannulus of $A$, then $A^{\prime}$ separates the boundary circles of $A$.

We define

$$
\begin{aligned}
\mathcal{A}(m) & :=\{A \mid A \text { is an Euclidean annulus with } \bmod (A)>m\}, \\
\mathcal{A}_{\Omega} & :=\{A \mid A \text { is an Euclidean annulus in } \Omega \text { with } c(A) \in \mathbb{C} \backslash \Omega\}, \\
\mathcal{A}_{\Omega}^{1} & :=\left\{A \in \mathcal{A}_{\Omega} \mid \partial A \cap \partial \Omega \neq \emptyset\right\}, \\
\mathcal{A}_{\Omega}^{2} & :=\left\{A \in \mathcal{A}_{\Omega} \mid \partial_{\text {in }} A \cap \partial \Omega \neq \emptyset \neq \partial_{\text {out }} A \cap \partial \Omega\right\}, \\
\mathcal{A}_{\Omega}(m) & :=\mathcal{A}_{\Omega} \cap \mathcal{A}(m), \text { and } \mathcal{A}_{\Omega}^{s}(m):=\mathcal{A}_{\Omega}^{s} \cap \mathcal{A}(m) \text { for } s \in\{1,2\} .
\end{aligned}
$$

The requirement $c(A) \in \mathbb{C} \backslash \Omega$ means that $A$ separates $(\mathbb{C} \backslash \Omega) \cup\{\infty\}$.

\footnotetext{
${ }^{7}$ Reflection across $\mathrm{S}^{1}(A)$ maps $A$ to itself interchanging its boundary circles.
} 
Following Pommerenke Pom79, we say that $E \subset \hat{\mathbb{C}}$ is $M$-uniformly perfect if and only if $E$ is closed, $E$ contains the point at infinity, and

$$
\sup _{A \in \mathcal{A}_{\mathbb{C} \backslash E}} \bmod (A) \leq M \text {. }
$$

Pommerenke Pom84] established a number of equivalent conditions. Heinonen Hei01 has a general metric space definition for uniform perfectness that is similar to, but different from, Pommerenke's definition; see the Appendix. See also Sug01] and HLM89].

2.C. Conformal metrics. A continuous function $X \stackrel{\rho}{\rightarrow}(0, \infty)$ on a rectifiably connected metric space $X$ induces a length distance $d_{\rho}$ on $X$ defined by

$$
d_{\rho}(a, b):=\inf _{\gamma: a \curvearrowright b} \ell_{\rho}(\gamma) \text { where } \ell_{\rho}(\gamma):=\int_{\gamma} \rho d s
$$

and where the infimum is taken over all rectifiable paths $\gamma: a \curvearrowright b$ in $X$. We describe this by calling $\rho d s=\rho(x)|d x|$ a conformal metric on $X$. Below we consider the hyperbolic and quasihyperbolic metrics defined on plane domains.

We call $\gamma$ a $\rho$-geodesic if $d_{\rho}(a, b)=\ell_{\rho}(\gamma)$; these need not be unique. We often write $[a, b]_{\rho}$ to indicate a $\rho$-geodesic with endpoints $a, b$, but one must be careful with this notation since these geodesics need not be unique. When $z$ is a fixed point on a given geodesic $[a, b]_{\rho}$, we write $[a, z]_{\rho}$ to mean the subarc of the given geodesic from $a$ to $z$.

We note that the ratio $\rho d s / \sigma d s$ of two conformal metrics is a well-defined positive function. We write $\rho \leq C \sigma$ to indicate that this metric ratio is bounded above by $C$.

When $\rho d s$ is a conformal metric on $\Omega$, we let $\Omega_{\rho}:=\left(\Omega, d_{\rho}\right)$. The following is surely folklore, but we briefly outline a proof which employs standard techniques.

2.3. Lemma. Let $\rho d s$ and $\tau d s$ be conformal metrics on some plane domain $\Omega$. Then the identity map

$$
\Omega_{\rho} \stackrel{\text { id }}{\rightarrow} \Omega_{\tau} \quad \text { is conformal, i.e., it is metrically 1-QC. }
$$

Also, if $\Omega_{\rho} \stackrel{f}{\rightarrow} \Omega_{\tau}$ is $\eta$-QS, then $f$ induces a map $\Omega \stackrel{f}{\rightarrow} \Omega$ which is $\eta(1)-Q C$.

Proof. The metric spaces $(\Omega,|\cdot|),(\Omega, l),\left(\Omega, d_{\rho}\right),\left(\Omega, d_{\tau}\right)$ are all homeomorphic, and even locally bi-Lipschitz. Indeed, given $a \in \Omega$ and $0<\varepsilon<\rho(a) / 2$, there is an $r_{0} \in(0, \delta(a))$ such that

$$
z \in \mathrm{D}\left[a ; r_{0}\right] \Longrightarrow(\rho(a)-\varepsilon)|z-a| \leq d_{\rho}(z, a) \leq(\rho(a)+\varepsilon)|z-a| .
$$

Using this we deduce that for all small $\varepsilon>0$ and all $r \in\left(0, r_{0}\right)$ (where $r_{0} \in(0, \delta(a)$ ) is chosen as above "for both $d_{\rho}$ and $d_{\tau}$ "),

$$
L(r):=\sup _{d_{\rho}(z, a) \leq r} d_{\tau}(z, a) \leq \frac{\tau(a)+\varepsilon}{\rho(a)-\varepsilon} \cdot r
$$

and

$$
l(r):=\inf _{d_{\rho}(z, a) \geq r} d_{\tau}(z, a) \geq \frac{\tau(a)-\varepsilon}{\rho(a)+\varepsilon} \cdot r
$$


whence

$$
\limsup _{r \rightarrow 0^{+}} \frac{L(r)}{l(r)} \leq \frac{\tau(a)+\varepsilon}{\tau(a)-\varepsilon} \cdot \frac{\rho(a)+\varepsilon}{\rho(a)-\varepsilon} \rightarrow 1 \text { as } \varepsilon \rightarrow 0^{+} .
$$

Suppose $\Omega_{\rho} \stackrel{f}{\rightarrow} \Omega_{\tau}$ is $\eta$-QS, $a \in \Omega$, and $\varepsilon>0$. Since $f$ is a homeomorphism, for all $r \in(0, \delta(a))$

$$
L_{f}(r):=\sup _{|z-a| \leq r}|f(z)-f(a)|=\max _{|z-a|=r}|f(z)-f(a)|
$$

and

$$
l_{f}(r):=\inf _{|z-a| \geq r}|f(z)-f(a)|=\min _{|z-a|=r}|f(z)-f(a)| .
$$

Below we write $z^{\prime}:=f(z), a^{\prime}:=f(a)$, etc.

As above, pick $r_{0} \in(0, \delta(a))$ and $s_{0} \in\left(0, \delta\left(a^{\prime}\right)\right)$ so that (2.4) holds for $d_{\rho}$ and its analog holds for $d_{\tau}$ and $w \in \mathrm{D}\left[a^{\prime} ; s_{0}\right]$. Then take $r_{1} \in\left(0, r_{0}\right]$ so that $f\left(\mathrm{D}\left[a ; r_{1}\right]\right) \subset$ $\mathrm{D}\left(a^{\prime} ; s_{0}\right)$. Then for all $r \in\left(0, r_{1}\right)$ and all $z, w \in \mathrm{S}^{1}(a ; r)$,

$$
\begin{aligned}
\frac{\left|z^{\prime}-a^{\prime}\right|}{\left|w^{\prime}-a^{\prime}\right|} & \leq \frac{\tau\left(a^{\prime}\right)+\varepsilon}{\tau\left(a^{\prime}\right)-\varepsilon} \cdot \frac{d_{\tau}\left(z^{\prime}, a^{\prime}\right)}{d_{\tau}\left(w^{\prime}, a^{\prime}\right)} \leq \frac{\tau\left(a^{\prime}\right)+\varepsilon}{\tau\left(a^{\prime}\right)-\varepsilon} \cdot \eta\left(\frac{d_{\tau}\left(z^{\prime}, a^{\prime}\right)}{d_{\tau}\left(w^{\prime}, a^{\prime}\right)}\right) \\
& \leq \frac{\tau\left(a^{\prime}\right)+\varepsilon}{\tau\left(a^{\prime}\right)-\varepsilon} \cdot \eta\left(\frac{\rho(a)+\varepsilon}{\rho(a)-\varepsilon}\right)
\end{aligned}
$$

here the inner inequality holds by quasisymmetry and the two outer inequalities follow from repeated applications of (2.4). Selecting such $z, w$ that attain $L_{f}(r), l_{f}(r)$ respectively yields

$$
\frac{L_{f}(r)}{l_{f}(r)}=\frac{\left|z^{\prime}-a^{\prime}\right|}{\left|w^{\prime}-a^{\prime}\right|} \leq \frac{\tau\left(a^{\prime}\right)+\varepsilon}{\tau\left(a^{\prime}\right)-\varepsilon} \cdot \eta\left(\frac{\rho(a)+\varepsilon}{\rho(a)-\varepsilon}\right)
$$

and so letting $r \rightarrow 0^{+}$, then $\varepsilon \rightarrow 0^{+}$, we deduce that, with respect to Euclidean distance, $f$ is indeed $\eta(1)$-QC.

The careful reader recognizes that in the above, we employ the metric (aka, linear) dilatation for quasiconformal maps, not the geometric dilatation, whereas in Fact 2.11 below $K$ is the geometric dilatation.

2.C.1. Quasihyperbolic metrics. The quasihyperbolic metric $\delta^{-1} d s$ is defined for any proper subdomain $\Omega \subsetneq \mathbb{C}$; here $\delta=\delta_{\Omega}$ is the Euclidean distance to the boundary of $\Omega$. This metric can be defined in very general metric spaces and has proven useful in many areas of geometric analysis. See [BHK01] and [HRS20].

For domains $\Omega \subsetneq \hat{\mathbb{C}}$, we also consider the chordal quasihyperbolic metric $\chi^{-1} d \hat{s}$ and the spherical quasihyperbolic metric $\sigma^{-1} d \hat{s}$ where $d \hat{s}$ denotes the chordal (or spherical) arclength "differential". (Recall the paragraph immediately preceding 2.A.) The latter was employed in [BHK01, Chapter 7].

The Euclidean, chordal, and spherical quasihyperbolic distances $k=k_{\Omega}, k_{\chi}=$ $k_{(\Omega, \chi)}$ and $k_{\sigma}=k_{(\Omega, \sigma)}$ in $\Omega$ are the length distances induced by the Euclidean quasihyperbolic, chordal quasihyperbolic, and spherical quasihyperbolic metrics $\delta^{-1} d s, \chi^{-1} d s$ and $\sigma^{-1} d s$ on $\Omega$; here $\Omega \subsetneq \mathbb{C}$ in the former setting whereas $\Omega \subsetneq \hat{\mathbb{C}}$ in the latter two. These are geodesic distances. 
The Euclidean length space $(\Omega, l)$ and spherical length space $\left(\Omega, l_{\sigma}\right)$ also carry quasihyperbolic metrics, but: quasihyperbolic distance in $(\Omega, l)$ is Euclidean quasihyperbolic distance and quasihyperbolic distance in $\left(\Omega, l_{\sigma}\right)$ is spherical quasihyperbolic distance.

We remind the reader of the following basic estimates for quasihyperbolic distance, first established by Gehring and Palka [GP76, 2.1]: For all $a, b \in \Omega$,

$$
k(a, b) \geq \log \left(1+\frac{l(a, b)}{\delta(a) \wedge \delta(b)}\right) \geq \log \left(1+\frac{|a-b|}{\delta(a) \wedge \delta(b)}\right) \geq\left|\log \frac{\delta(a)}{\delta(b)}\right|
$$

where $l(a, b)$ is the (intrinsic) length distance between $a$ and $b$. The first inequality above is a special case of the more general (and easily proven) inequality

$$
\ell_{k}(\gamma) \geq \log \left(1+\frac{\ell(\gamma)}{\operatorname{dist}(|\gamma|, \partial \Omega)}\right)
$$

which holds for any rectifiable path $\gamma$ in $\Omega$. See also [BHK01, (2.3),(2.4)]. There are analogous inequalities for $k_{\chi}$ and $k_{\sigma}$ where we replace all the Euclidean metric quantities by the appropriate chordal or spherical metric quantities.

It is well known that the holomorphic covering $\mathbb{C} \stackrel{\exp }{\longrightarrow} \mathbb{C}_{\star}$ pulls back the quasihyperbolic metric $\delta_{\star}^{-1} d s$ on $\mathbb{C}_{\star}$ to the Euclidean metric on $\mathbb{C}$, which in turn reveals that $\left(\mathbb{C}_{\star}, k_{\star}\right)$ is (isometric to) the Euclidean cylinder $\mathbb{S}^{1} \times \mathbb{R}^{1}$ with its Euclidean length distance inherited from its standard embedding into $\mathbb{R}^{3}$; here $k_{\star}:=k_{\mathbb{C}_{\star}}$. See MO86]. In particular, quasihyperbolic geodesics in $\mathbb{C}_{\star}$ are logarithmic spirals and for all $a, b \in \mathbb{C}_{\star}$,

$$
k_{\star}(a, b)=|\log (b / a)|=|\log | b / a|+i \operatorname{Arg}(b / a)|
$$

and thus

$$
\left|\log \frac{|b|}{|a|}\right| \vee\left|\operatorname{Arg}\left(\frac{b}{a}\right)\right| \leq k_{\star}(a, b) \leq\left|\log \frac{|b|}{|a|}\right|+\left|\operatorname{Arg}\left(\frac{b}{a}\right)\right| \leq\left|\log \frac{|b|}{|a|}\right|+\frac{\pi}{2} \frac{|a-b|}{|a| \wedge|b|} .
$$

Note the special cases of the above that arise when $|a|=|b|$ or $\operatorname{Arg}(b / a)=0$.

Often, (2.6) provides good estimates for quasihyperbolic distances as described next.

2.7. Fact. Suppose $A=\{r<|z-c|<R\} \subset \Omega$ with $c \in \mathbb{C} \backslash \Omega$ and $R / r>4$. Let $\delta_{*}^{-1} d s$ and $k_{*}$ denote the quasihyperbolic metric and distance in the punctured plane $\mathbb{C} \backslash\{c\}$. Then in $\{2 r<|z-c|<R / 2\}, \frac{1}{2} \delta_{*} \leq \delta \leq \delta_{*}$ and $k_{*} \leq k \leq 2 k_{*}$.

Another consequence of (2.6) is the following description for the two 'ends' of $\left(\mathbb{C}_{\star}, k_{\star}\right)$. We include this trivial observation as motivation for later results; see Lemma 3.12 and Proposition 3.13

2.8. Lemma. Let $r \in(0,+\infty)$. Define $\Delta_{\star}, \Delta^{\star} \subset \mathbb{C}_{\star}$ by

$$
\Delta_{\star}:=\mathrm{D}[0 ; r] \backslash\{0\} \quad \text { and } \quad \Delta^{\star}:=\mathbb{C} \backslash \mathrm{D}(0 ; r) .
$$

Then both $\left(\Delta_{\star}, k_{\star}\right)$ and $\left(\Delta^{\star}, k_{\star}\right)$ are $\pi$-roughly isometrically equivalent to the infinite ray $([0,+\infty),|\cdot|)$. 
Proof. It is easy to see that the map $\left(\Delta_{\star}, k_{\star}\right) \stackrel{\varphi}{\rightarrow}([0,+\infty),|\cdot|), \varphi(z):=\log (|z| / r)$, is a surjective $(1, \pi)$-QI equivalence; also, here $\vartheta(s):=r e^{-s}$ is an isometric embedding from $([0,+\infty),|\cdot|)$ into $\left(\Delta_{\star}, k_{\star}\right)$ with

$$
\vartheta([0,+\infty))=(0, r] \subset \Delta_{\star} \subset N_{k_{\star}}[(0, r] ; \pi] .
$$

For $\left(\Delta^{\star}, k_{\star}\right)$, we use the fact that $z \mapsto z^{-1}$ is an isometric automorphism of $\left(\mathbb{C}_{\star}, k_{\star}\right)$.

As the chordal and spherical distances are bi-Lipschitz equivalent, and $\sigma$ is the length distance associated with $\chi$ (on $\hat{\mathbb{C}}$ ), it follows that the chordal and spherical quasihyperbolic metrics (and their associated distances) are bi-Lipschitz equivalent with $k_{\sigma} \leq k_{\chi} \leq \frac{\pi}{2} k_{\sigma}$.

It is useful to know that Euclidean and spherical quasihyperbolic distances are bi-Lipschitz equivalent. Note, however, that the distortion constant depends on the location of the origin. Essentially, this is because a general Möbius transformation is not a chordal nor spherical isometry, just bi-Lipschitz. One can establish this by using appropriate estimates between $\delta$ and $\chi$ as in [BHX08, Lemma 3.10] or alternatively appeal to [BHX08, Theorem 4.12]; the interested reader should also peruse BB03.

2.9. Fact. Let $\Omega \subsetneq \mathbb{C}$ be a domain. Then $(\Omega, k),\left(\Omega, k_{\chi}\right)$, and $\left(\Omega, k_{\sigma}\right)$ are all biLipschitz equivalent. In particular, $\frac{1}{4} k \leq k_{\chi} \leq 8(1+D)^{4} k$ where $D:=\operatorname{dist}(0, \mathbb{C} \backslash \Omega) 8$

An important property of hyperbolic distance is its conformal invariance. While this does not hold for quasihyperbolic distance, it is Möbius quasi-invariant in the following sense; see [GP76, Lemma 2.4, Corollary 2.5].

2.10. Fact. Let $\hat{\mathbb{C}} \stackrel{T}{\rightarrow} \hat{\mathbb{C}}$ be a Möbius transformation. Suppose $\Omega \subsetneq \mathbb{C}$ and $\Omega^{\prime}:=$ $T(\Omega) \subset \mathbb{C}$. Then $(\Omega, k) \stackrel{T}{\rightarrow}\left(\Omega^{\prime}, k^{\prime}\right)$ is 2-bilipschitz, where $k^{\prime}:=k_{\Omega^{\prime}}$.

In GO79, Theorem 3], Gehring and Osgood proved the following, which says that quasiconformal homeomorphisms are rough quasihyperbolic quasiisometries and conformal maps are even quasihyperbolically bi-Lipschitz.

2.11. Fact. For each $K \geq 1$ there is a constant $C=C(K)$ such that for any $K$-QC homeomorphism $f: \Omega \rightarrow \Omega^{\prime}$ between two proper plane domains,

$$
\forall a, b \in \Omega, \quad k^{\prime}(f(a), f(b)) \leq C \max \left\{k(a, b), k(a, b)^{p}\right\}
$$

where $p:=K^{-1}$. In particular, a conformal map between $\Omega$ and $\Omega^{\prime}$ induces a bi-Lipschitz equivalence between $(\Omega, k)$ and $\left(\Omega^{\prime}, k^{\prime}\right)$.

2.C.2. Hyperbolic metrics. Every hyperbolic domain in $\hat{\mathbb{C}}$ carries a unique metric, $\lambda d s=\lambda_{\Omega} d s$, which enjoys the property that its pullback $p^{*}[\lambda d s]$, with respect to any holomorphic universal covering projection $p: \mathbb{D} \rightarrow \Omega$, is the hyperbolic metric $\lambda_{\mathbb{D}}(\zeta)|d \zeta|=2\left(1-|\zeta|^{2}\right)^{-1}|d \zeta|$ on $\mathbb{D}$. Another description is that $\lambda d s$ is the unique maximal (or unique complete) metric on $\Omega$ that has constant Gaussian curvature -1 . In terms of such a covering $p$, the metric-density $\lambda=\lambda_{\Omega}$ of the Poincaré hyperbolic metric $\lambda_{\Omega} d s$ can be determined from

$$
\lambda(z)=\lambda_{\Omega}(z)=\lambda_{\Omega}(p(\zeta))=2\left(1-|\zeta|^{2}\right)^{-1}\left|p^{\prime}(\zeta)\right|^{-1},
$$

\footnotetext{
${ }^{8}$ One should view the constant $D$ as depending on $\operatorname{diam}_{\chi} \Omega$. By examining the distances between 0 and 1 in $\mathrm{D}(0 ; R)$ (or in $\mathbb{C} \backslash\{R\}$ ) (as $R \rightarrow+\infty$ ) we see that this bi-Lipschitz constant really does depend on $D$.
} 
the above being valid for points $z \in \Omega \cap \mathbb{C}$ whereas one must use local coordinates in any neighborhood of the point at infinity if $\Omega \not \subset \mathbb{C}$. (Alternatively, one can use the chordal hyperbolic metric-density $\hat{\lambda}$ and then the hyperbolic metric is $\hat{\lambda} \hat{d} s$ where $\hat{d s}$ denotes the chordal (or spherical) arclength "differential".)

For example, the hyperbolic metric $\lambda_{*} d s$ on the punctured unit disk $\mathbb{D}_{*}:=\mathbb{D} \backslash\{0\}$ can be obtained by using the universal covering $z=\exp (w)$ from the left-half-plane onto $\mathbb{D}_{*}$ and we find that

$$
\lambda_{*}(z)|d z|=\frac{|d z|}{|z||\log | z||} .
$$

The hyperbolic distance $h=h_{\Omega}$ is the length distance $h_{\Omega}:=d_{\lambda}$ induced by the hyperbolic metric $\lambda d s$ on $\Omega$. This is a geodesic distance: for any points $a, b$ in $\Omega$, there is an $h$-geodesic $[a, b]_{h}$ joining $a, b$ in $\Omega$. These geodesics need not be unique, but they enjoy the property that

$$
h(a, b)=\ell_{h}\left([a, b]_{h}\right) .
$$

Here we are writing $\ell_{h}$ in lieu of $\ell_{\lambda}$.

Except for a short list of special cases, the actual calculation of any given hyperbolic metric is notoriously difficult; computing hyperbolic distances and determining hyperbolic geodesics is even harder. Indeed, one can find a number of papers analyzing the behavior of the hyperbolic metric in a twice punctured plane. Typically one is left with estimates obtained by using domain monotonicity and considering 'nice' sub-domains and super-domains in which one can calculate, or at least estimate, the metric.

The standard technique for estimating the hyperbolic metric and hyperbolic distance is via domain monotonicity, a consequence of Schwarz's Lemma. That is, if $\Omega_{\text {in }} \subset \Omega \subset \Omega_{\text {out }}$, then in $\Omega_{\text {in }}, \lambda_{\text {in }} d s \geq \lambda d s \geq \lambda_{\text {out }} d s$ and $h_{\text {in }} \geq h \geq h_{\text {out }}$.

Notice that the largest hyperbolic plane regions are twice punctured planes. We write $\lambda_{a b} d s$ and $h_{a b}$ for the hyperbolic metric and hyperbolic distance in the twice punctured plane $\mathbb{C}_{a b}$. The standard twice punctured plane is $\mathbb{C}_{01}$ and its hyperbolic metric has been extensively studied by numerous researchers including Hem79], Min87, SV01, SV05]. We mention only the following.

2.12. Fact. For all $z \in \mathbb{C}_{01}, \lambda_{01}(z) \geq \lambda_{01}(-|z|) \geq(|z|[k+|\log | z||])^{-1}$, with equality at $z$ if and only if $z=-1$. Here $\mathrm{k}:=\left(\lambda_{01}(-1)\right)^{-1}=\Gamma^{4}(1 / 4) /\left(4 \pi^{2}\right)=$ $4.3768796 \ldots$

Fact 2.12 was first proved by Lehto, Virtanen and Väisälä (see [LVV59]); later proofs were given by Agard Aga68, Jenkins [Jen81, and Minda [Min87.

For future reference we record the following well-known estimates for hyperbolic distance in $\mathbb{D}_{\star}$, in $\mathbb{D}^{\star}$, and in $\mathbb{C}_{01}$. Since we know the hyperbolic metrics in $\mathbb{D}_{\star}$ and $\mathbb{D}^{\star}$, it is easy to check the first two estimates. The estimates for $h_{01}$ are straightforward consequences of Fact 2.12 above.

\subsection{Facts.}

(a) For any $a, b \in \mathbb{D}_{\star}, h_{\star}(a, b) \leq\left|\log \frac{\log (1 /|a|)}{\log (1 /|b|)}\right|+\frac{\pi}{\log 2}$.

(b) For any $a, b \in \mathbb{D}^{\star}, h^{\star}(a, b) \leq\left|\log \frac{\log |a|}{\log |b|}\right|+\frac{\pi}{\log 2}$. 
(c) For all $a, b \in \mathbb{C}_{01}$ :

$$
1 \leq|a| \leq|b| \Longrightarrow h_{01}(a, b) \geq h_{01}(-|a|,-|b|) \geq \log \frac{\mathrm{k}+\log |b|}{\mathrm{k}+\log |a|}
$$

and

$$
|a| \leq|b| \leq 1 \Longrightarrow h_{01}(a, b) \geq h_{01}(-|a|,-|b|) \geq \log \frac{\mathrm{k}+\log (1 /|a|)}{\mathrm{k}+\log (1 /|b|)} .
$$

2.C.3. The Beardon-Pommerenke function bp. We desire upper and lower estimates for the hyperbolic metric in terms of the quasihyperbolic metric. These metrics are 2-bi-Lipschitz equivalent for simply connected hyperbolic plane regions; this is false for any domain with an isolated boundary point (such as the punctured unit disk). The hyperbolic and quasihyperbolic metrics are bi-Lipschitz equivalent precisely when $\hat{\mathbb{C}} \backslash \Omega$ is uniformly perfect (see BP78, Pom79, Pom84). Beardon and Pommerenke corroborated this latter assertion as an application of their elegant result [BP78, Theorem 1] which says:

For any hyperbolic region $\Omega$ in $\mathbb{C}$ and for all $z \in \Omega$,

$$
\frac{1}{\delta(z)(\mathrm{k}+\mathrm{bp}(z))} \leq \lambda(z) \leq \frac{\pi / 2}{\delta(z) \mathrm{bp}(z)} .
$$

Here the domain function $\Omega \stackrel{\text { bp }}{\longrightarrow} \mathbb{R}$, introduced by Beardon and Pommerenke, is defined via

$$
\operatorname{bp}(z)=\operatorname{bp}_{\Omega}(z):=\inf _{\substack{\zeta \in B(z) \\ \xi \in(\mathbb{C} \backslash \Omega) \backslash\{\zeta\}}}|\log | \frac{\zeta-z}{\zeta-\xi}|| ;
$$

note that the infimum is restricted to nearest boundary points $\zeta \in \mathrm{B}(z)=\partial \Omega \cap$ $\partial D(z)$ for $z$, that is, $\zeta \in \partial \Omega$ with $\delta(z)=|z-\zeta|$.

The definition of $\mathrm{bp}$ is motivated by examining the standard lower bound for the hyperbolic metric on a twice punctured plane. The (BP) inequalities follow via domain monotonicity: the upper bound for $\lambda(z)$ holds because $z$ lies on the conformal center of a certain annulus in $\Omega$, and the lower bound holds because $\Omega$ lies in a certain twice punctured plane.

A geometric interpretation for $\mathrm{bp}(z)$ is seen by defining $\mathrm{bp}(z, \zeta)$, for $z \in \Omega$ and $\zeta \in \mathrm{B}(z)$, as

$$
\operatorname{bp}(z, \zeta):=\inf _{\xi \in(\mathbb{C} \backslash \Omega) \backslash\{\zeta\}}|\log | \frac{\zeta-z}{\zeta-\xi}|| . \quad\left(\text { Thus, } \mathrm{bp}(z)=\inf _{\zeta \in \mathrm{B}(z)} \mathrm{bp}(z, \zeta)\right) .
$$

Then $2 \mathrm{bp}(z, \zeta)$ is the conformal modulus of the maximal Euclidean annulus that is contained in $\Omega$ and symmetric with respect to the circle $S^{1}(\zeta ; \delta(z))$. It follows that $2 \mathrm{bp}(z)$ is the minimum of these numbers; so, $2 \mathrm{bp}(z)$ is the smallest of these maximal moduli.

Thus we see that whenever $\operatorname{bp}(z)>0$, there is an annulus

$$
\mathrm{BP}(z):=\mathrm{A}(\zeta ; \delta(z), \operatorname{bp}(z)) \in \mathcal{A}_{\Omega}^{1} \quad(\text { so, } \operatorname{BP}(z) \subset \Omega \text { and } \partial \mathrm{BP}(z) \cap \partial \Omega \neq \emptyset)
$$

associated with $z$; here $\zeta \in \mathrm{B}(z)$ is any nearest boundary point for $z$ that realizes $\operatorname{bp}(z)$, and

$$
\forall \xi \in \partial \operatorname{BP}(z) \cap \partial \Omega, \quad \operatorname{bp}(z)=\operatorname{bp}(z, \zeta)=|\log | \frac{\zeta-z}{\zeta-\xi}|| .
$$


We call $\operatorname{BP}(z)$ a Beardon-Pommerenke annulus (or briefly, a BP annulus) associated with the point $z$; it needn't be unique.

Typically, the Beardon-Pommerenke inequalities (BP) are employed to give lower estimates for hyperbolic distance, but they can also provide useful upper estimates.

2.14. Example. Let $0<a<b$ with $\log (b / a)>2$. Put $\Omega:=\mathbb{C} \backslash\{0,-a,-b\}$. Then

$$
h(a, b) \leq 4+\pi \log \left(\frac{1}{2} \log \frac{b}{a}\right) .
$$

Proof. Let $s:=\sqrt{a b}$. Evidently, for $x \in[a, b], \delta(x)=x$ and

$$
\mathrm{bp}(x)=\left|\log \frac{x}{a}\right| \wedge\left|\log \frac{b}{x}\right| \text {. }
$$

Thus by (BP)

$$
h(a e, s) \leq \frac{\pi}{2} \int_{a e}^{s} \frac{d x}{x \log (x / a)}=\frac{\pi}{2} \log \log \left(\frac{b}{a}\right)^{1 / 2}
$$

and similarly, $h(s, b / e) \leq \frac{\pi}{2} \log \log (b / a)^{1 / 2}$. Hence

$$
h(a, b) \leq 2 k(a, a e)+h(a e, b / e)+2 k(b / e, b) \leq 4+\pi \log \left(\frac{1}{2} \log \frac{b}{a}\right)
$$

as claimed.

In [BH20, Proposition 3.3] we established especially useful estimates for bp; these say that in any annulus in $\mathcal{A}_{\Omega}^{2}(8 \log 2)$, the domain function bp decays 'linearly' as we move away from the center circle. The assumption that both boundary circles of the annulus meet $\partial \Omega$ is crucial for obtaining the upper bounds; when only one boundary circle has a boundary point, bp can actually increase when we move away from the center circle towards the boundary circle that does not meet $\partial \Omega$. Here is a summary.

2.15. Fact. Let $\mathrm{A}(o ; d, r) \in \mathcal{A}_{\Omega}^{2}(8 \log 2)$, so $r>\log 16$. For $|t| \lesssim r$ and all $z \in$ $\mathrm{S}^{1}\left(o ; d e^{t}\right), \operatorname{bp}(z) \simeq r-|t|$. More precisely, for $|t| \leq r-\log 16$ and $z \in \mathrm{S}^{1}\left(o ; d e^{t}\right)$,

$$
\frac{1}{2}(r-|t|) \leq \mathrm{bp}(z) \leq 2(r-|t|) .
$$

In the above, $z \in \mathrm{A}[o ; d, r-\log 16]$ and $t:=\log (|z-o| / d)$. In particular:

(a) In $\mathrm{A}[o ; d, r-\log 16]$, bp $\leq 2 r=\bmod (A)$, and on $\mathrm{S}^{1}(A)=\mathrm{S}^{1}(o ; d)$, bp $\geq \frac{1}{2} r$.

(b) On both boundary circles $\mathrm{S}^{1}\left(o ; 16^{ \pm 1} d e^{\mp r}\right), 2 \log 2 \leq$ bp $\leq 8 \log 2$.

(c) For $\log 16<q<r$ we have the strict inequalities

$$
\mathrm{bp}>\frac{1}{2} q \text { in } \mathrm{A}(o ; d, r-q) \quad \text { and } \quad \mathrm{bp}<2 q \text { in } \mathrm{A}(o ; d, r-\log 16) \backslash \mathrm{A}[o ; d, r-q] .
$$

(d) We also have estimates for bp in annuli $A=\mathrm{A}(o ; d, r) \in \mathcal{A}_{\Omega}^{1}$, as long as we move towards the boundary circle that has a boundary point. 
2.C.4. The $A B C$ property. A path $\mathbb{R} \supset I \stackrel{\gamma}{\rightarrow} \Omega$ has the arcs bounce or cross property with parameters $\mu>0$ and $\nu>0$, abbreviated as the $(\mu, \nu)-A B C$ property, if and only if for each compact subpath $\alpha$ of $\gamma$ and for each annulus $A:=\mathrm{A}(o ; d, \nu) \in \mathcal{A}_{\Omega}$ such that $\mathrm{S}^{1}(A) \supset \partial \alpha$, we have $|\alpha| \subset \mathrm{A}(o ; d, \mu)$.

The ABC property implies that the path can cross a moderate size annulus at most once, so if the path enters deep into an annulus, it either stays there or it crosses (once) and never returns. In particular, if the path goes near an "isolated island or archipelago" of $\Omega^{c} \cup\{\infty\}$, then it stays near it. There are various precise statements of this phenomenon given in [BH20, Lemma 3.7].

We utilize the following; see [BH20, Lemma 3.7(a), Prop. 3.8].

\subsection{Facts.}

(a) Suppose $\mathbb{R} \supset I \stackrel{\gamma}{\rightarrow} \Omega$ has the $A B C$ property with parameters $\mu \geq \nu>0$. If $\mathrm{A}(o ; d, \nu) \in \mathcal{A}_{\Omega}$, then $\gamma$ crosses $\mathrm{A}(o ; d, \mu)$ at most once.

(b) Quasihyperbolic geodesics have the $(\pi, \log 2)$-ABC property,

(c) Hyperbolic geodesics have the $(3 \mathrm{k}, 5 / 2)$-ABC property.

2.D. Gromov hyperbolicity and uniformity. Thanks to the ground-breaking work in BHK01], we know that Gromov hyperbolicity and uniformity are intimately connected.

Roughly speaking, a metric space is uniform when points in it can be joined by paths which are not "too long" and which "move away" from the region's boundary. More precisely, $\Omega \subset \mathbb{C}$ is $C$-uniform (for some constant $C \geq 1$ ) provided each pair of points can be joined by a $C$-uniform arc. Here a rectifiable arc $\gamma: a \curvearrowright b$ is a $C$-uniform arc if and only if it is both a $C$-quasiconvex arc and a double $C$-cone arc; these conditions mean, respectively, that

$$
\ell(\gamma) \leq C|a-b|
$$

and

$$
\forall z \in|\gamma|, \quad \ell(\gamma[z, a]) \wedge \ell(\gamma[z, b]) \leq C \delta(z) .
$$

Martio and Sarvas introduced the notion of a uniform domain in MS79, and this has proven to be invaluable in geometric function theory and especially for the "analysis in metric spaces" program. A simply connected proper subdomain of the plane is uniform if and only if it is a quasidisk. Each uniform domain has the Sobolev extension property, and the BMO extension property characterizes uniformity. See [Geh82] and the many references therein, especially [Jon81, Jon80].

A geodesic metric space $X$ is Gromov hyperbolic if there exists a constant $\theta \geq 0$ such that every geodesic triangle is $\theta$-thin, meaning that each point on any edge of the triangle is at distance at most $\theta$ from the other two edges. See BHK01, Chapter 3], BH99, BBI01, or [Väi05] and the many references in these.

The Gromov boundary $\partial_{G} X$ of a Gromov hyperbolic space $X$ is the set of equivalence classes of geodesic rays, where two rays are equivalent if and only if their Hausdorff distance is finite. One can also use quasi-geodesic rays, or, Gromov sequences. There is no canonical preferred distance on the Gromov boundary. However, for each $\varepsilon \in\left(0, \varepsilon_{0}\right]$ (usually $\varepsilon_{0}=\varepsilon_{0}(\theta):=1 \wedge(1 / 5 \theta)$ ) there is a so-called visual distance $d_{\varepsilon}=d_{\varepsilon, o}$ on $\partial_{G} X$ that satisfies

$$
\frac{1}{2} \exp \left(-\varepsilon(\xi \mid \eta)_{o}\right) \leq d_{\varepsilon}(\xi, \eta) \leq \exp \left(-\varepsilon(\xi \mid \eta)_{o}\right)
$$


for all $\xi, \eta \in \partial_{G} X$, where $(\xi \mid \eta)_{o}$ is the usual Gromov product and $o \in X$ a fixed base point. Standard estimates then give

$$
C^{-1} \exp (-\varepsilon \operatorname{dist}(o,(\xi, \eta))) \leq d_{\varepsilon}(\xi, \eta) \leq C \exp (-\varepsilon \operatorname{dist}(o,(\xi, \eta)))
$$

where $(\xi, \eta)$ is any geodesic line in $X$ with endpoints $\xi, \eta \in \partial_{G} X$.

The conformal gauge on $\partial_{G} X$ is the maximal collection of all distance functions on $\partial_{G} X$ that are quasisymmetrically equivalent to some (hence all) visual distance(s).

\section{Proofs of theOREMS}

3.A. Proof of Theorem A. We employ the following technical fact about "fat" annuli.

3.1. Lemma. Suppose $m:=\frac{1}{2} \bmod (A)>1$ for some annulus $A \subset \Omega$ with center in $\mathbb{C} \backslash \Omega$ and $\partial A \cap \partial \Omega \neq \emptyset$. Then there are points $a, b, c \in A$ with:

$$
\frac{1}{2}(m-1) \leq k \leq m-1, \quad \text { for } k \in\{k(a, b), k(c, b)\},
$$

and

$$
h(a, b) \geq \log \left(1+\frac{m-1}{2(\mathrm{k}+1)}\right), \quad \text { whereas } h(c, b) \leq 1.1 .
$$

Proof. By similarity invariance we may assume $A=\left\{z: e^{-m}<|z|<e^{m}\right\}$ and either $-e^{-m} \in \partial \Omega$ or $-e^{m} \in \partial \Omega$; here $m>1$. Assume $-e^{-m} \in \partial \Omega$ and define

$$
a:=e^{1-m}, \quad b:=\sqrt{a}, \quad c:=1 .
$$

We demonstrate that these points possess the asserted properties.

Thanks to Fact 2.7 we know that $k_{\star} \leq k \leq 2 k_{\star}$ in $A$. Since

$$
2 k_{\star}(a, b)=2 \log \frac{|b|}{|a|}=\log \frac{1}{|a|}=m-1=2 k_{\star}(c, b),
$$

the inequalities in (3.1a) follow.

Since $\Omega \subset \mathbb{C} \backslash\left\{0,-e^{-m}\right\}$, an appeal to Fact 2.13(c) provides the estimate

$$
\begin{aligned}
h(a, b) & \geq h_{-e^{-m} 0}(a, b)=h_{01}\left(-e^{m} a,-e^{m} b\right) \\
& \geq \log \frac{\mathrm{k}+\log \left(e^{m} b\right)}{\mathrm{k}+\log \left(e^{m} a\right)}=\log \left(1+\frac{m-1}{2(\mathrm{k}+1)}\right) .
\end{aligned}
$$

Employing (BP) we deduce that

$$
\begin{aligned}
h(c, b) & \leq \int_{[b, c]} \lambda d s \leq \frac{\pi}{2} \int_{[b, c]} \frac{d s}{\delta \mathrm{bp}} \\
& =\frac{\pi}{2} \int_{b}^{c} \frac{d t}{t(m+\log t)}=\frac{\pi}{2} \log \frac{2 m}{m+1}<1.1 .
\end{aligned}
$$

When $-e^{m} \in \partial \Omega$ we take $a:=e^{m-1}, b:=\sqrt{a}, c:=1$ and argue similarly.

3.2. Proof of Theorem [A] Thanks to the work BP78] of Beardon and Pommerenke, it suffices to explain why $(\mathrm{A} 1)$ implies $(2.2)$, but perhaps it is illuminating to see why (A.2) implies (2.2); these implications are both quantitative. 
With this in mind, suppose $(\Omega, h) \stackrel{f}{\rightarrow}(\Omega, k)$ is $K$-bi-Lipschitz. Let $A \in \mathcal{A}_{\Omega}$, and let $\alpha$ be the simple loop in $\Omega$ whose trajectory is the center circle $|\alpha|=\mathrm{S}^{1}(A)$. Using hyperbolic distance in $A \subset \Omega$, we deduce that

$$
\ell_{h}(\alpha) \leq 2 \pi^{2} / \bmod (A) \text {. }
$$

Since $f \circ \alpha$ is an essential loop in $\Omega$, we obtain

$$
2 \pi \leq \ell_{k}(f \circ \alpha) \leq K \ell_{h}(\alpha) \leq 2 K \pi^{2} / \bmod (A)
$$

and thus $\bmod (A) \leq \pi K$.

Now suppose $(\Omega, h) \stackrel{f}{\rightarrow}(\Omega, k)$ is $\eta$-QS. Thanks to Lemma 2.3 we know that $\Omega \stackrel{f}{\rightarrow} \Omega$ is $K$-QC where $K=K(\eta(1))$ depends only on the value $\eta(1)$. Let $C=$ $C(K)=C(\eta(1))$ be the constant from Fact 2.11. We establish (2.2) with the upper bound

$$
M:=\max \left\{4 C+2,2+4(\mathrm{k}+1) \exp \left(1.1\left(\eta^{-1}\left(1 / 2 C^{2}\right)\right)^{-1}\right)\right\} .
$$

Let $A \in \mathcal{A}_{\Omega}$. By enlarging $A$ if necessary, we may assume that $\partial A \cap \partial \Omega \neq \emptyset$, and also that $m:=\frac{1}{2} \bmod (A)>2 C+1$. Let $a, b, c$ be the points in $A$ given by Lemma 3.1 and let $a^{\prime}, b^{\prime}, c^{\prime}$ be their $f$ images. Since $k(a, b) \geq \frac{1}{2}(m-1) \geq 1$, Fact 2.11 tells us that

$$
k\left(a^{\prime}, b^{\prime}\right) \leq C k(a, b) .
$$

The same fact, now applied to $f^{-1}$, tells us that

$$
C \max \left\{k^{\prime}\left(c^{\prime}, b^{\prime}\right), k^{\prime}\left(c^{\prime}, b^{\prime}\right)^{p}\right\} \geq k(c, b) \geq \frac{1}{2}(m-1) \geq C,
$$

so $k^{\prime}\left(c^{\prime}, b^{\prime}\right) \geq 1$ whence $k(c, b) \leq C k^{\prime}\left(c^{\prime}, b^{\prime}\right)$. Therefore

$$
k^{\prime}\left(a^{\prime}, b^{\prime}\right) \leq C k(a, b) \leq 2 C k(c, b) \leq 2 C^{2} k^{\prime}\left(c^{\prime}, b^{\prime}\right)
$$

and thus by quasisymmetry

$$
\frac{1}{2 C^{2}} \leq \frac{k^{\prime}\left({ }^{\prime} c^{\prime}, b^{\prime}\right)}{k^{\prime}\left(a^{\prime}, b^{\prime}\right)} \leq \eta\left(\frac{h(c, b)}{h(a, b)}\right) \leq \eta\left(1.1\left(\log \left(1+\frac{m-1}{2(\mathrm{k}+1)}\right)^{-1}\right)\right.
$$

which gives the asserted estimate $2 m \leq M$.

3.B. Proof of Theorem B. It seems plausible, especially in light of Proposition $3.4(\mathrm{~g})$ below (and its quasihyperbolic analog), that one could give a direct proof of Theorem B. The authors are unable to do so; instead, we base our proof on the following hyperbolic analog of [BHK01, Theorem 3.6]. Note that it depends heavily on BHK01, Theorems 1.11, 1.12, and Prop. 7.12] and these in turn depend on the Bonk-Heinonen-Koskela uniformization theory. Our proof also utilizes the fact $[\mathrm{BH} 20$, Theorem $\mathrm{A}]$ that hyperbolic and quasihyperbolic quasi-geodesics are the same curves; in particular, in uniform domains hyperbolic geodesics are uniform arcs [Her21b, Remarks 4.3].

3.3. Theorem. Let $\Omega$ be a hyperbolic domain in $\hat{\mathbb{C}}$. Suppose $\left(\Omega, l_{\sigma}\right)$ is uniform. Then the canonical conformal gauge on $\partial_{G}(\Omega, h)$ is naturally guasisymmetrically equivalent to the conformal gauge on $\partial\left(\Omega, l_{\sigma}\right)$ determined by $l_{\sigma} 9$

\footnotetext{
${ }^{9}$ Recall that here $l_{\sigma}$ denotes the intrinsic length distance in $(\Omega, \sigma)$.
} 
The above is quantitative, but the constants are somewhat murky!

Bonk, Heinonen, and Koskela established a similar result [BHK01, Theorem 3.6] for abstract uniform metric spaces but using quasihyperbolic distance in lieu of hyperbolic distance. We closely follow their proof, but there are significant modifications that we detail.

In particular, we utilize the following information; much of this is either a direct consequence of work in BHK01, or follows by similar reasoning, the latter being especially true whenever only upper estimates for quasihyperbolic distance are employed (because always, $h \leq 2 k$ ). See especially BHK01, Chapters 2 and 3]. We sketch the ideas.

Below, and later, when $(\Omega, h)$ is Gromov hyperbolic (in which case $(\Omega, k)$ is also Gromov hyperbolic), we write $h_{\varepsilon}=h_{\varepsilon, o}$ and $k_{\varepsilon}=k_{\varepsilon, o}$ for the standard visual distances on $\partial_{G}(\Omega, h)$ and $\partial_{G}(\Omega, k)$ repectively; here, as in $\$ 2 . \mathrm{D}$, the visual parameter $\varepsilon \in\left(0, \varepsilon_{0}\right]$ and $o \in \Omega$ is a fixed base point.

3.4. Proposition. Let $\Omega$ be a hyperbolic domain in $\hat{\mathbb{C}}$ with $\left(\Omega, l_{\sigma}\right)$ A-uniform. There are constants $\theta, B, C$ (that depend only on the "data") such that the following hold.

(a) The metric space $(\Omega, h)$ is Gromov $\theta$-hyperbolic.

(b) There is $o \in \Omega$ with $\sigma(o)=\max _{\Omega} \sigma$ and $2 \leq \operatorname{diam}\left(\Omega, l_{\sigma}\right) / \sigma(o) \leq 2 A$, so $\operatorname{diam}\left(\Omega, l_{\sigma}\right) \leq 2 \pi A$.

(c) Each pair of distinct points in $\overline{\left(\Omega, l_{\sigma}\right)}$ can be joined by a hyperbolic geodesic that is a B-uniform arc in $\left(\Omega, l_{\sigma}\right)$; when one or both points lie in $\partial\left(\Omega, l_{\sigma}\right)$, we get a hyperbolic geodesic ray or line respectively.

(d) There is a natural bijection between $\partial_{G}(\Omega, h)$ and $\partial\left(\Omega, l_{\sigma}\right)$ given by $[\gamma] \mapsto \gamma(\infty)$ where $[\gamma] \in \partial_{G}(\Omega, h)$ is the equivalence class of a hyperbolic geodesic ray $\gamma$ that has the endpoint $\gamma(\infty) \in \partial\left(\Omega, l_{\sigma}\right)$. Therefore, we use the same notation for points in $\partial_{G}(\Omega, h)$ or $\partial\left(\Omega, l_{\sigma}\right)$.

(e) Given $\zeta \in \partial\left(\Omega, l_{\sigma}\right)$ and a hyperbolic geodesic ray $[o, \zeta)_{h}$ in $\Omega$, each $\xi \in \partial\left(\Omega, l_{\sigma}\right)$ has an associated point $x=x(\xi) \in[o, \zeta)_{h}$ with

$$
h\left(x,(\xi, \zeta)_{h}\right) \leq C
$$

and

$$
h(o, x)-C \leq h\left(o,(\xi, \zeta)_{h}\right) \leq h(o, x)+C .
$$

In fact, if $\gamma$ is the $\sigma$-arclength parametrization for $[o, \zeta]_{h}$, starting at $\gamma(0)=\zeta$, then we can take

$$
x:= \begin{cases}\gamma\left(l_{\sigma}(\xi, \zeta)\right) & \text { when } l_{\sigma}(\xi, \zeta) \leq \frac{1}{2} l_{\sigma}(o, \zeta) \\ o & \text { otherwise. }\end{cases}
$$

(f) Given $\zeta, \xi \in \partial\left(\Omega, l_{\sigma}\right)$ and $x=x(\xi) \in[o, \zeta)_{h}$ as above, we have

$$
C^{-1} e^{-\varepsilon h(x, o)} \leq h_{\varepsilon}(\zeta, \xi) \leq C e^{-\varepsilon h(x, o)} .
$$

Proof. There is no harm in rotating the sphere $\hat{\mathbb{C}}$, so we can assume that $\Omega \subset$ $\mathbb{C}$. To see (a), we start with the fact (see [BHK01, Theorem 3.6]) that $\left(\Omega, k_{\sigma}\right)$ is $\theta$-hyperbolic with $\theta=\theta(A)$, so by Fact $2.9(\Omega, k)$ is $\theta$-hyperbolic with $\theta=$ $\theta(A, \operatorname{dist}(0, \mathbb{C} \backslash \Omega))$. Then $[\mathrm{BH} 20$, Theorem B] tells us that $(\Omega, h)$ is also $\theta$-hyperbolic with $\theta=\theta(A, \operatorname{dist}(0, \mathbb{C} \backslash \Omega))$. 
Part (b) is elementary. For (c), we note that by [BH20, Theorem A], geodesic segments in $(\Omega, h)$ are quasi-geodesics in $(\Omega, k)$ (with an absolute constant) and hence by Fact 2.9 are also quasi-geodesics in $\left(\Omega, k_{\sigma}\right)$ now with a constant that depends on $\operatorname{dist}(0, \mathbb{C} \backslash \Omega)$. Finally, Her21b, Theorem 4.1] affirms that these arcs are $B$-uniform with $B=B(A, d)$. The assertions about geodesic rays and lines that end at boundary points follow in standard ways as explained in BHK01, Proposition 3.12].

Item (e) can be established exactly as done in [BHK01, Lemma 3.14] for quasihyperbolic distance. Evidently, (f) follows from (e) and the standard estimates for visual distances given at the end of $₫ 2 . \mathrm{D}$.

Item (d) follows mostly as in [BHK01, Proposition 3.12] with one major modification. It is routine to see that each hyperbolic geodesic ray in $\Omega$ has an endpoint in $\partial\left(\Omega, l_{\sigma}\right)$, that rays with the same endpoint are equivalent, and that each boundary point is the endpoint of such a ray. It remains to show that equivalent rays have the same endpoint. Suppose $\alpha$ and $\beta$ are hyperbolic geodesic rays in $\Omega$ with $\xi:=\alpha(\infty), \eta:=\beta(\infty) \in \partial\left(\Omega, l_{\sigma}\right)$, and $\xi \neq \eta$. We claim that $\operatorname{dist}_{\mathcal{H}}^{h}(|\alpha|,|\beta|)=+\infty$ (so $\alpha$ and $\beta$ are not equivalent).

This is not difficult to check when $\xi$ and $\eta$ correspond te 10 different points in $\hat{\partial} \Omega=\partial(\Omega, \sigma)$, but requires additional effort if these two length boundary points are attached to the same spherical boundary point, which we assume is the origin 0. Since we are "near" the origin, we can work with Euclidean quantities in place of spherical.

As in [BHK01, Proposition 3.12], since $\xi \neq \eta$, there is a quasihyperbolic geodesic line $\gamma=(\xi, \eta)_{k}$ in $\Omega$. Let $\Lambda:=\ell(\gamma)$ and let $z_{o}$ be the Euclidean arclength midpoint of $\gamma$. Then

$$
\left|z_{o}\right| \geq \delta\left(z_{o}\right) \geq \frac{\Lambda}{2 B}
$$

Put $L:=\min \{\Lambda / 10 B, \ell(\alpha), \ell(\beta)\}$. Pick $s_{a}<s_{o}<s_{b}$ so that $z_{o}=\gamma\left(s_{o}\right)$ and and so the quasihyperbolic subrays $\gamma_{a}(s):=\gamma\left(s_{a}-s\right), \gamma_{b}(s):=\gamma\left(s_{b}+s\right)($ for $s \in[0,+\infty))$ of $\gamma$ both have length

$$
\ell\left(\gamma_{a}\right)=L=\ell\left(\gamma_{b}\right)
$$

By trimming the initial parts of $\alpha, \beta$ (if necessary), we may assume they both have length $L$. Let $a:=\gamma\left(s_{a}\right), b:=\gamma\left(s_{b}\right)$ be the initial points of $\gamma_{a}, \gamma_{b}$ respectively.

Note that $\gamma_{a}, \gamma_{b}$ are quasihyperbolic subrays of $\gamma$ with $\gamma_{a}(\infty)=\xi, \gamma_{b}(\infty)=\eta$. As $\gamma$ is also a hyperbolic quasi-geodesic line in $\Omega$ (by [BH20, Theorem A]) and $\alpha(\infty)=\gamma_{a}(\infty), \beta(\infty)=\gamma_{b}(\infty)$, there is a finite constant $H$ such that

$$
\forall s \geq 0, \quad h\left(\alpha(s), \gamma_{a}(s)\right) \leq H \quad \text { and } \quad h\left(\beta(s), \gamma_{b}(s)\right) \leq H .
$$

Since $\xi \neq \eta$, there is "plenty" of $\partial \Omega$ "near" the origin. In particular, it is not hard to check that bp $\leq \log 10$ on $\left|\gamma_{a}\right| \cup\left|\gamma_{b}\right|$, so by (BP) $\lambda d s$ and $\delta^{-1} d s$ are bi-Lipschitz on $\left|\gamma_{a}\right| \cup\left|\gamma_{b}\right|$. It follows that for all $s>\left|s_{a}\right| \vee\left|s_{b}\right|$,

$$
\ell_{h}\left(\gamma\left[-s, s_{a}\right]\right) \simeq \ell_{k}\left(\gamma\left[-s, s_{a}\right]\right)=k(\gamma(-s), a)
$$

\footnotetext{
${ }^{10}$ The identity map $\left(\Omega, l_{\sigma}\right) \stackrel{\text { id }}{\rightarrow}(\Omega, \sigma)$ is 1-Lipschitz, so has a 1-Lipschitz extension to a map $\overline{\left(\Omega, l_{\sigma}\right)} \stackrel{\iota}{\rightarrow}(\hat{\Omega}, \sigma)$ and the $\iota$ image of $\partial\left(\Omega, l_{\sigma}\right)$ is precisely the set of rectifiably accessible boundary points of $(\Omega, \sigma)$; see Her10 Prop. 3.22].)
} 
and

$$
\ell_{h}\left(\gamma\left[s_{b}, s\right]\right) \simeq \ell_{k}\left(\gamma\left[s_{b}, s\right]\right)=k(\gamma(s), b),
$$

so $\ell_{h}(\gamma[-s, s]) \gtrsim k(\gamma(-s), a)+k(\gamma(s), b)$. Finally, for all sufficiently large $s>0$,

$$
\begin{aligned}
h(\alpha(s), \beta(s)) & \geq h(\gamma(-s), \gamma(s))-h(\alpha(s), \gamma(-s))-h(\beta(s), \gamma(s)) \\
& \geq h(\gamma(-s), \gamma(s))-2 H \gtrsim \ell_{h}(\gamma[-s, s])-2 H \\
& \gtrsim k(\gamma(-s), a)+k(\gamma(s), b)-2 H \rightarrow+\infty(\text { as } s \rightarrow \infty) .
\end{aligned}
$$

Thus $\alpha$ and $\beta$ are indeed non-equivalent hyperbolic geodesic rays :-)

We require the following technical information. The upshot of this is that, given two length boundary points, we can always find spherical boundary points at a distance comparable to the length distance between the two given length boundary points. Here $\iota$ is as described in footnote 10. Also, we employ the ABC property for hyperbolic geodesics; see $\$ 2 . C .4$.

3.5. Lemma. Let $\mathrm{c}:=e^{-(\mu+\nu)}$ where $\mu, \nu$ are $A B C$ parameters for hyperbolic distance 11 Let $\Omega \subset \hat{\mathbb{C}}$ be a hyperbolic domain. Suppose $\xi, \eta$ are distinct points in $\partial\left(\Omega, l_{\sigma}\right)$. Assume there is a hyperbolic geodesic line $\gamma:=(\xi, \eta)_{h}$ that is also a $B$ uniform arc in $\left(\Omega, l_{\sigma}\right)$. Let $z_{0}$ be the $\sigma$-arclength midpoint of $\gamma$. Put $\xi_{0}:=\iota(\xi), \eta_{0}:=$ $\iota(\eta)$, and $r_{0}:=\chi\left(z_{0}, \eta_{0}\right)$. Then

$$
\text { either } \quad \chi\left(\xi_{0}, \eta_{0}\right) \geq r_{0}, \quad \text { or } \quad \hat{\partial} \Omega \cap\left\{z \in \hat{\mathbb{C}} \mid \mathrm{c} r_{0} \leq \chi\left(z, \eta_{0}\right) \leq r_{0}\right\} \neq \emptyset .
$$

Thus there exists a point $\xi_{1} \in \hat{\partial} \Omega$ such that $\chi\left(\xi_{1}, \eta_{0}\right) \simeq l_{\sigma}(\xi, \eta)$; more precisely,

$$
\frac{\mathrm{c}}{\pi B} l_{\sigma}(\xi, \eta) \leq \chi\left(\xi_{1}, \eta_{0}\right) \leq \frac{B}{2} l_{\sigma}(\xi, \eta) .
$$

Proof. We will see, after some normalization, that (3.5a) follows directly from the $\mathrm{ABC}$ property and then $(3.5 \mathrm{~b})$ is an easy consequence of uniformity. To verify (3.5a), assume $\chi\left(\xi_{0}, \eta_{0}\right)<\mathrm{cr}$. By rotating $\hat{\mathbb{C}}$ if necessary, we may assume that $\eta_{0}=0 \in \mathbb{C}$.

Note that

$$
f(t):=\frac{t}{\sqrt{4-t^{2}}} \quad \text { has } f(\chi(z, 0))=|z|,
$$

so $f$ is increasing on $[0,2)$; also,

$$
f^{-1}(s)=\frac{2 s}{\sqrt{1+s^{2}}}
$$

Thus $f\left(r_{0}\right)=f\left(\chi\left(z_{0}, \eta_{0}\right)\right)=\left|z_{0}\right|$ and for any $r \in\left(0, r_{0}\right)$,

$$
A:=\left\{z \mid r<\chi\left(z, \eta_{0}\right)<r_{0}\right\}=\left\{z|f(r)<| z \mid<f\left(r_{0}\right)\right\} .
$$

We set $r:=f^{-1}\left(\mathrm{c}\left|z_{0}\right|\right)$, so: $f(r)=\mathrm{c}\left|z_{0}\right|, A=\left\{\mathrm{c}\left|z_{0}\right|<|z|<\left|z_{0}\right|\right\}$, and $\bmod (A)=$ $\mu+\nu$. Also, note that

$$
r=f^{-1}\left(\mathrm{c}\left|z_{0}\right|\right)=\frac{2 \mathrm{c}\left|z_{0}\right|}{\sqrt{1+\left(\mathrm{c}\left|z_{0}\right|\right)^{2}}}>\frac{2 \mathrm{c}\left|z_{0}\right|}{\sqrt{1+\left|z_{0}\right|^{2}}}=\mathrm{c} r_{0}>\chi\left(\xi_{0}, \eta_{0}\right)=f^{-1}\left(\left|\xi_{0}\right|\right) .
$$

\footnotetext{
${ }^{11}$ We take $\mu:=3 \mathrm{k}$ and $\nu:=5 / 2$; then $\mu>\nu$ and $\mathrm{c}$ is an absolute constant.
} 
So $\left|\xi_{0}\right|<\mathrm{c}\left|z_{0}\right|$. This gives $A \cap \partial \Omega \neq \emptyset$, as we explain below, and then (3.5a) follows.

To this end, notice that as $\left|\xi_{0}\right|<\mathrm{c}\left|z_{0}\right|$, there is a subarc $\alpha$ of $\gamma$ with $z_{0} \in|\alpha|$ and $\partial \alpha \subset \mathrm{S}^{1}\left(0 ; e^{-\mu}\left|z_{0}\right|\right)$. Since $\mu>\nu, \mathrm{A}\left(0 ; e^{-\mu}\left|z_{0}\right|, \nu\right) \subset A$. If $A \subset \Omega$ were true, then by employing the fact that $\gamma$ enjoys the $(\mu, \nu)$-ABC property (see 2. C.4 $)$ we could assert that $|\alpha| \subset \mathrm{A}\left(0 ; e^{-\mu}, \mu\right)=A$; but, $z_{0} \in|\alpha|$ and $z_{0} \notin A$. Therefore, $A \cap \partial \Omega \neq \emptyset$, so (3.5a) holds.

Now we establish (3.5b). As $\gamma$ is a $B$-uniform arc in $\left(\Omega, l_{\sigma}\right)$,

$$
l_{\sigma}(\xi, \eta) \leq \ell_{\sigma}(\gamma) \leq B l_{\sigma}(\xi, \eta) .
$$

Also,

$$
r_{0}=\chi\left(z_{0}, \eta_{0}\right) \leq \ell_{\sigma}\left(\gamma\left[z_{0}, \eta\right]\right)=\frac{1}{2} \ell_{\sigma}(\gamma) \leq \frac{B}{2} l_{\sigma}(\xi, \eta)
$$

and

$$
r_{0} \geq \chi\left(z_{0}\right) \geq \frac{2}{\pi} \sigma\left(z_{0}\right) \geq \frac{2}{\pi B} \ell_{\sigma}\left(\gamma\left[z_{0}, \eta\right]\right) \geq \frac{1}{\pi B} l_{\sigma}(\xi, \eta)
$$

Thus if $\chi\left(\xi_{0}, \eta_{0}\right) \geq r_{0}$, then

$$
\frac{1}{\pi B} l_{\sigma}(\xi, \eta) \leq r_{0} \leq \chi\left(\xi_{0}, \eta_{0}\right) \leq \sigma\left(\xi_{0}, \eta_{0}\right) \leq l_{\sigma}(\xi, \eta)
$$

and (3.5b) holds with $\xi_{1}:=\xi_{0}$. Suppose $\chi\left(\xi_{0}, \eta_{0}\right)<r_{0}$. Then by (3.5a) there is a point $\xi_{1} \in \hat{\partial} \Omega$ with

$$
\frac{\mathrm{c}}{\pi B} l_{\sigma}(\xi, \eta) \leq c r_{0} \leq \chi\left(\xi_{1}, \eta_{0}\right) \leq r_{0} \leq \frac{B}{2} l_{\sigma}(\xi, \eta)
$$

as asserted in (3.5b).

Armed with the notation and results from Proposition 3.4 and Lemma 3.5, we now establish Theorem 3.3 .

3.6. Proof of Theorem 3.3 . We may assume $\Omega \subset \mathbb{C}$. Then $\left(\Omega, k_{\sigma}\right) \stackrel{\text { id }}{\rightarrow}(\Omega, k)$ is biLipschitz as explained in Fact 2.9, Let $\mathrm{c}:=e^{-(\mu+\nu)}$ be the constant in Lemma 3.5.

As in [BHK01, Theorem 3.6], we show that the bijection $\partial\left(\Omega, l_{\sigma}\right) \rightarrow \partial_{G}(\Omega, h)$ (given in Proposition 3.4(d)) is a quasisymmetry; here we assume $h_{\varepsilon}$ is a standard visual distance on $\partial_{G}(\Omega, h)$ as in $\$ 2$.D with visual parameter $\varepsilon \in\left(0, \varepsilon_{0}\right]$ and $o \in \Omega$ is a fixed base point as given in Proposition 3.4(b).

Let $\zeta, \eta, \xi$ be points in $\partial\left(\Omega, l_{\sigma}\right)$ and put $t:=l_{\sigma}(\zeta, \xi) / l_{\sigma}(\zeta, \eta)$. When $t \geq 1$, we can copy the Bonk-Heinonen-Koskela argument as it only uses upper estimates for quasihyperbolic distances 12 Thus we may, and do, assume that $t<1$.

Let $x=x(\xi), y=y(\eta)$ be the points on the hyperbolic geodesic ray $[o, \zeta)_{h}$ that are given by Proposition [3.4(e) and associated with $\xi, \eta$ respectively. Since $t<1$, we have $\zeta<x \leq y \leq o$ where the geodesic is ordered from $\zeta$ to $o$. Then from Proposition 3.4(f) we find that

$$
\frac{h_{\varepsilon}(\zeta, \xi)}{h_{\varepsilon}(\zeta, \eta)} \leq C e^{-\varepsilon h(x, y)} \leq C .
$$

\footnotetext{
${ }^{12}$ Here we use the fact that $h \leq 2 k \leq 8 k_{\sigma}$.
} 
It follows that for any fixed $t_{0} \in(0,1)$,

$$
\forall t \in\left[t_{0}, 1\right), \quad \frac{h_{\varepsilon}(\zeta, \xi)}{h_{\varepsilon}(\zeta, \eta)} \leq \frac{C}{t_{0}} t
$$

To finish the proof, we demonstrate that for all $0<t<t_{0}:=\mathrm{c} / \pi B^{2}, h(x, y) \geq H(t)$ where $H(t) \rightarrow+\infty$ as $t \rightarrow 0^{+}$. This then gives

$$
\frac{h_{\varepsilon}(\zeta, \xi)}{h_{\varepsilon}(\zeta, \eta)} \leq C e^{-\varepsilon H(t)} \rightarrow 0 \text { as } t \rightarrow 0^{+}
$$

which in turn confirms that the bijection $\partial\left(\Omega, l_{\sigma}\right) \rightarrow \partial_{G}(\Omega, h)$ is indeed quasisymmetric.

Before immersing ourselves in the details, we explain the idea. Whenever one knows three distinct boundary points, one has a standard lower bound for hyperbolic distance given by looking at the appropriate thrice punctured sphere; Fact 2.13 (c) is handy for estimating hyperbolic distance in such a domain. A difficulty here is that we have points in $\partial\left(\Omega, l_{\sigma}\right)$ whereas we need points in $\hat{\partial} \Omega$. To overcome this, we appeal to Lemma 3.5

With this in mind, let $\zeta_{0}:=\iota(\zeta), \xi_{0}:=\iota(\xi), \eta_{0}:=\iota(\eta)$; see footnote 10. By Lemma 3.5 there are points $\xi_{1}, \eta_{1} \in \hat{\partial} \Omega$ with $\xi_{1} \neq \eta_{1}$ (when $t<t_{0}$ ) and such that

$$
\frac{\mathrm{c}}{\pi B} l_{\sigma}(\xi, \zeta) \leq \chi\left(\xi_{1}, \zeta_{0}\right) \leq \frac{B}{2} l_{\sigma}(\xi, \zeta)
$$

and

$$
\frac{\mathrm{c}}{\pi B} l_{\sigma}(\eta, \zeta) \leq \chi\left(\eta_{1}, \zeta_{0}\right) \leq \frac{B}{2} l_{\sigma}(\eta, \zeta) .
$$

Let $x^{\prime}:=T(x), y^{\prime}:=T(y)$ denote the images of $x, y$ (respectively) under the Möbius transformation $T$ that maps $\zeta_{0}, \xi_{1}, \eta_{1}$ to $0,1, \infty$ respectively; so

$$
T(z):=\left[z, \zeta_{0}, \eta_{1}, \xi_{1}\right]=\frac{\left(z-\zeta_{0}\right)\left(\eta_{1}-\xi_{1}\right)}{\left(z-\eta_{1}\right)\left(\zeta_{0}-\xi_{1}\right)} .
$$

Since $\Omega \subset \Omega_{0}:=\hat{\mathbb{C}} \backslash\left\{\zeta_{0}, \xi_{1}, \eta_{1}\right\}$, writing $h_{0}:=h_{\Omega_{0}}$, we now have

$$
h(x, y) \geq h_{0}(x, y)=h_{01}\left(x^{\prime}, y^{\prime}\right) \geq h_{01}\left(-\left|x^{\prime}\right|,-\left|y^{\prime}\right|\right) .
$$

To complete the proof, we demonstrate below that

$$
\text { as } t \rightarrow 0^{+}, \quad\left|x^{\prime}\right| \simeq 1 \text { and }\left|y^{\prime}\right| \simeq \frac{1}{t}
$$

which, in conjunction with Fact 2.13(c), provides the desired estimate.

First we show that $1 \simeq\left|x^{\prime}\right|=\left|x, \zeta_{0}, \eta_{1}, \xi_{1}\right|=\frac{\chi\left(x, \zeta_{0}\right) \chi\left(\eta_{1}, \xi_{1}\right)}{\chi\left(x, \eta_{1}\right) \chi\left(\zeta_{0}, \xi_{1}\right)}$. We claim that

$$
\frac{4}{\pi B^{2}} \chi\left(\xi_{1}, \zeta_{0}\right) \leq \chi\left(x, \zeta_{0}\right) \leq \frac{\pi B}{\mathrm{c}} \chi\left(\xi_{1}, \zeta_{0}\right), \quad \text { so } \quad \frac{\chi\left(x, \zeta_{0}\right)}{\chi\left(\zeta_{0}, \xi_{1}\right)} \leq \frac{\pi B}{\mathrm{c}} .
$$

To see this, we use the definition of $x:=\gamma\left(l_{\sigma}(\xi, \zeta)\right)$ along with (3.7a) to obtain

$$
\chi\left(x, \zeta_{0}\right) \leq \sigma\left(x, \zeta_{0}\right) \leq \ell_{\sigma}(\gamma[x, \zeta])=l_{\sigma}(\xi, \zeta) \leq \frac{\pi B}{\mathrm{c}} \chi\left(\xi_{1}, \zeta_{0}\right)
$$


and also, as $\zeta_{0} \in \hat{\partial} \Omega$ and $\gamma$ is $B$-uniform in $\left(\Omega, l_{\sigma}\right)$,

$$
\frac{\pi}{2} \chi\left(x, \zeta_{0}\right) \geq \sigma\left(x, \zeta_{0}\right) \geq \sigma(x) \geq B^{-1} \ell_{\sigma}(\gamma[x, \zeta])=B^{-1} l_{\sigma}(\xi, \zeta) \geq \frac{2}{B^{2}} \chi\left(\xi_{1}, \zeta_{0}\right) .
$$

Next, continuing to use the inequalities (3.7) we deduce that

$$
\begin{aligned}
\chi\left(\xi_{1}, \eta_{1}\right) & \leq \chi\left(\zeta_{0}, \xi_{1}\right)+\chi\left(\zeta_{0}, \eta_{1}\right) \\
& \leq \frac{B}{2}\left(l_{\sigma}(\zeta, \xi)+l_{\sigma}(\zeta, \eta)\right)=\frac{B}{2}(1+t) l_{\sigma}(\zeta, \eta) \leq B l_{\sigma}(\zeta, \eta)
\end{aligned}
$$

and, when $t \leq t_{0}=\mathrm{c} / \pi B^{2}$,

$$
\begin{aligned}
\chi\left(\xi_{1}, \eta_{1}\right) & \geq \chi\left(\zeta_{0}, \eta_{1}\right)-\chi\left(\zeta_{0}, \xi_{1}\right) \geq \frac{\mathrm{c}}{\pi B} l_{\sigma}(\zeta, \eta)-\frac{B}{2} l_{\sigma}(\zeta, \xi) \\
& =\left(\frac{\mathrm{c}}{\pi B}-\frac{B}{2} t\right) l_{\sigma}(\zeta, \eta) \geq \frac{\mathrm{c}}{2 \pi B} l_{\sigma}(\zeta, \eta) .
\end{aligned}
$$

From the above inequalities we see that, when $t \leq t_{0}$,

$$
\frac{\mathrm{c}}{2 \pi B} l_{\sigma}(\zeta, \eta) \leq \chi\left(\xi_{1}, \eta_{1}\right) \leq B l_{\sigma}(\zeta, \eta) .
$$

Replacing $\xi_{1}$ with $x$ and repeating the argument directly above, we check that when $t \leq t_{1}:=\mathrm{c} / 2 \pi B$,

$$
\frac{\mathrm{c}}{2 \pi B} l_{\sigma}(\zeta, \eta) \leq \chi\left(x, \eta_{1}\right) \leq B l_{\sigma}(\zeta, \eta) .
$$

Combining the above we now find that when $t \leq t_{0}<t_{1}$,

$$
\frac{\chi\left(\xi_{1}, \eta_{1}\right)}{\chi\left(x, \eta_{1}\right)} \leq \frac{B l_{\sigma}(\zeta, \eta)}{(\mathrm{c} / 2 \pi B) l_{\sigma}(\zeta, \eta)}=\frac{2 \pi}{\mathrm{c}} B^{2}
$$

Finally,

$$
\left|x^{\prime}\right|=\frac{\chi\left(x, \zeta_{0}\right) \chi\left(\eta_{1}, \xi_{1}\right)}{\chi\left(x, \eta_{1}\right) \chi\left(\zeta_{0}, \xi_{1}\right)} \leq \frac{\pi B}{\mathrm{c}} \frac{2 \pi}{\mathrm{c}} B^{2}=\frac{2 \pi^{2}}{\mathrm{c}^{2}} B^{3} .
$$

It remains to explain why $\frac{1}{t} \simeq\left|y^{\prime}\right|=\frac{\chi\left(y, \zeta_{0}\right) \chi\left(\eta_{1}, \xi_{1}\right)}{\chi\left(y, \eta_{1}\right) \chi\left(\zeta_{0}, \xi_{1}\right)}$. From above, we already know that

$$
\frac{\mathrm{c}}{\pi B^{2}} \frac{1}{t}=\frac{(\mathrm{c} / 2 \pi B) l_{\sigma}(\zeta, \eta)}{(B / 2) l_{\sigma}(\xi, \zeta)} \leq \frac{\chi\left(\eta_{1}, \xi_{1}\right)}{\chi\left(\zeta_{0}, \xi_{1}\right)} \leq \frac{B l_{\sigma}(\zeta, \eta)}{(\mathrm{c} / \pi B) l_{\sigma}(\xi, \zeta)}=\frac{\pi B^{2}}{\mathrm{c}} \frac{1}{t}
$$

when $t \leq t_{0}$. Thus it suffices to demonstrate that $\chi\left(y, \zeta_{0}\right) \simeq \chi\left(y, \eta_{1}\right)$. There are two cases depending on whether or not $y=o$. When $y=o$ we find that

$$
\frac{1}{\pi A} \leq \frac{\chi\left(y, \zeta_{0}\right)}{\chi\left(y, \eta_{1}\right)} \leq \pi A
$$

the diligent reader can confirm this with the help of Proposition 3.4(b). We assume $y \neq o$, or equivalently, $l_{\sigma}(\zeta, \eta) \leq \frac{1}{2} l_{\sigma}(\eta, o)$. Here $y=\gamma\left(l_{\sigma}(\eta, \zeta)\right)$, so $\chi\left(y, \zeta_{0}\right) \leq l_{\sigma}(y, \zeta) \leq \ell_{\sigma}(\gamma[y, \zeta])=l_{\sigma}(\eta, \zeta) \quad$ and $\quad \chi\left(y, \eta_{1}\right) \leq \chi\left(y, \zeta_{0}\right)+\chi\left(\eta_{1}, \zeta_{0}\right) \leq 2 l_{\sigma}(\eta, \zeta)$ and then by uniformity

$$
\chi\left(y, \zeta_{0}\right) \wedge \chi\left(y, \eta_{1}\right) \geq \chi(y) \geq \frac{2}{\pi} \sigma(y) \geq \frac{2}{\pi B} l_{\sigma}(\eta, \zeta)
$$


whence

$$
\frac{1}{\pi B} \leq \frac{\chi\left(y, \zeta_{0}\right)}{\chi\left(y, \eta_{1}\right)} \leq \frac{\pi}{2} B
$$

3.8. Proof of Theorem B. Since Euclidean translations are isometries of both $(\Omega, h)$ and $(\Omega, k)$, there is no harm in assuming that the origin lies in $\partial \Omega$. In this setting, $(\Omega, k)$ and $\left(\Omega, k_{\sigma}\right)$ are bi-Lipschitz equivalent with an absolute constant. It now follows that $(\Omega, h),(\Omega, k)$ and $\left(\Omega, k_{\sigma}\right)$ are all Gromov hyperbolic (or not, but we assume the former).

It is well known (see [Gol69, Theorem 1, p.211] or [Tsu75, Theorem IX.22]) that $\Omega$ is conformally equivalent to a horizontal slit domain $\Omega^{\prime} \subsetneq \hat{\mathbb{C}}$; thus $\infty \in \Omega^{\prime}$ and each boundary component of $\Omega^{\prime}$ is either a point or a compact horizontal line segment. One can show that $\left(\Omega^{\prime}, l_{\sigma}^{\prime}\right)$ is $C$-LLC ${ }_{2}$ with $C:=\pi / \sigma^{\prime}(\infty)$. Therefore by [BHK01, Prop.7.12], $\left(\Omega^{\prime}, l_{\sigma}\right)$ is uniform.

We are now positioned to apply Theorem 3.3 and its quasihyperbolic analog [BHK01, Theorem 3.6]. These two results provide the first two QS equivalences $\cong$ below:

$$
\partial_{G}(\Omega, h) \equiv \partial_{G}\left(\Omega^{\prime}, h^{\prime}\right) \cong \partial\left(\Omega, l_{\sigma}\right) \cong \partial_{G}\left(\Omega^{\prime}, k_{\sigma}^{\prime}\right) \cong \partial_{G}(\Omega, k) ;
$$

the isometric equivalence $\equiv$ holds because conformal maps are hyperbolic isometries, and the last QS equivalence $\cong$ holds because $\left(\Omega^{\prime}, k_{\sigma}^{\prime}\right)$ and $(\Omega, k)$ are biLipschitz equivalent thanks to Fact 2.11

3.C. Proof of Theorem C. Below, in 3.C.3, we establish the following general result; this provides a large class of plane domains whose hyperbolizations and quasihyperbolizations are quasiisometrically equivalent. In particular, each finitely connected domain belongs to this class, thus corroborating Theorem C. However, this class also includes many infinitely connected domains such as $\mathbb{C} \backslash \mathbb{Z}$ or $\mathbb{C}_{\star} \backslash\left\{\frac{1}{n} \mid\right.$ $n \in \mathbb{N}\}$.

3.9. Theorem. Let $\Pi \subset \Omega \subset \hat{\mathbb{C}}$ be as described in 3. C.1 with (3.10) holding. In addition, suppose that

$$
\hat{\mathbb{C}} \backslash \Omega_{\Delta}=(\hat{\mathbb{C}} \backslash \Omega) \cup \Delta \quad \text { is } M \text {-uniformly perfect. }
$$

Then $\left(\Omega_{\Pi}, h_{\Pi}\right)$ and $\left(\Omega_{\Pi}, k_{\Pi}\right)$ are $(L, 6 \pi)$-quasiisometrically equivalent where $L=$ $L(M)$.

3.C.1. Notation and key assumptions. Let $\Pi \subset \Omega \subset \hat{\mathbb{C}}$ and put $\Omega_{\Pi}:=\Omega \backslash \Pi$. Here $\Omega$ can be $\mathbb{C}$ or even $\hat{\mathbb{C}}$, but we assume that $\Pi$ is closed in $\Omega$, that $\Omega_{\Pi} \subset \mathbb{C}$, and that $\Omega_{\Pi}$ is hyperbolic; in particular, the point at infinity belongs to $\Omega$ if and only if it belongs to $\Pi$. For convenience, we set $\Pi^{\star}:=\Pi \backslash\{\infty\}$.

We assume that for each $p \in \Pi$ there is an associated $r_{p}>0$ with the properties described below. For $p \neq \infty$, we set

$$
\Delta_{p}:=\mathrm{D}\left[p ; r_{p}\right] \text { and } \Delta_{p}^{\star}:=\Delta_{p} \backslash\{p\},
$$

and we assume that

$$
2 r_{p} \leq \delta(p) \quad\left(\text { so } \mathrm{D}\left(p ; 2 r_{p}\right) \subset \Omega\right)
$$


and that for points $p \neq q$ in $\Pi^{\star}$,

$$
2\left(r_{p}+r_{q}\right) \leq|p-q| \quad\left(\text { so } \mathrm{D}\left(p ; 2 r_{p}\right) \cap \mathrm{D}\left(q ; 2 r_{q}\right)=\emptyset\right) .
$$

To handle finitely connected domains, e.g., $\mathbb{D}^{\star}$ or $\mathbb{C}_{01}$, we must allow $\infty \in \Pi$ in which case we further assume that

$$
\hat{\mathbb{C}} \backslash \Omega \subset \mathrm{D}\left[0 ; \frac{1}{4} r_{\infty}\right] \quad \text { and that } \quad \Delta_{p} \subset \mathrm{D}\left[0 ; \frac{1}{4} r_{\infty}\right] \text { for each } p \in \Pi^{\star} .
$$

When $\infty \in \Pi$, we set

$$
\Delta_{\infty}:=\hat{\mathbb{C}} \backslash \mathrm{D}\left(0 ; r_{\infty}\right) \text { and } \quad \Delta_{\infty}^{\star}:=\Delta_{\infty} \backslash\{\infty\}
$$

Next, define

$$
\Delta:=\bigcup_{p \in \Pi} \Delta_{p} \quad \text { and } \quad \Omega_{\Delta}:=\Omega \backslash \Delta .
$$

In the above, $\delta=\delta_{\Omega}$ is the Euclidean distance to $\partial \Omega$ which is infinite if $\Omega \supset \mathbb{C}$.

The hypotheses above ensure that $\Pi$ is discrete in $\Omega$, so $\Omega_{\Pi}$ is a domain. Similarly, $\Delta$ is closed in $\Omega$ and $\Omega_{\Delta}$ is a domain. We use the subscripts $\Pi$ and $\Delta$ to denote quantities associated with $\Omega_{\Pi}$ and $\Omega_{\Delta}$. For example, $h_{\Pi}=h_{\Omega_{\Pi}}$ and $k_{\Pi}=k_{\Omega_{\Pi}}$ are the hyperbolic and quasihyperbolic distances in $\Omega_{\Pi}$ (respectively), and especially $\mathrm{bp}_{\Pi}=\mathrm{bp}_{\Omega_{\Pi}}$.

3.C.2. Quasiisometric equivalence of punctured disks. Here we present some technical details that allow us to streamline our proof of Theorem 3.9 and focus on the underlying ideas. Roughly, we show that whenever $\Delta_{*}$ is a punctured disk in a hyperbolic plane domain $\Omega$ (with the puncture a point of $\hat{\mathbb{C}} \backslash \Omega$ ), $\left(\Delta_{*}, h\right)$ and $\left(\Delta_{*}, k\right)$ are quasiisometrically equivalent.

We use the following numerical result whose proof is left for the diligent reader.

$$
\forall K>0, L>0, x>0, y \geq L: \quad \frac{K+x}{K+y} \geq \frac{L}{K+L} \frac{x}{y} .
$$

We require a result similar to Lemma 2.8 but for punctured disks in arbitrary domains; here there are separate cases for finite versus infinite punctures. Since Euclidean similarity transformations are quasihyperbolic isometries, we can normalize as in the following.

3.12. Lemma. Suppose $\mathbb{D} \subset \Omega \subset \mathbb{C}$ with $1 \in \mathbb{C} \backslash \Omega$. Let $\Omega_{*}:=\Omega \backslash\{0\}$ and $k_{*}:=k_{\Omega_{*}}$. For each $r \in\left(0, \frac{1}{2}\right],\left(\Delta_{*}, k_{*}\right)$ is $\pi$-roughly isometrically equivalent to the infinite ray $([0,+\infty),|\cdot|)$, where $\Delta_{*}:=\mathrm{D}[0 ; r] \backslash\{0\}$.

Next, suppose $0 \in \hat{\mathbb{C}} \backslash \Omega \subset \overline{\mathbb{D}}$. Let $\Omega^{*}:=\Omega \backslash\{\infty\}$ and $k^{*}:=k_{\Omega^{*}}$. For each $R \geq$ $2,\left(\Delta^{*}, k^{*}\right)$ is $(2, \pi)$-quasiisometrically equivalent to the infinite ray $([0,+\infty),|\cdot|)$, where $\Delta^{*}:=\mathbb{C} \backslash \mathrm{D}(0 ; R)$.

Proof. In $\left\{0<|z|<\frac{1}{2}\right\} \supset \Delta_{*}, \delta_{*}(z)=|z|=\delta_{\star}(z)$, so the identity map

$$
\left(\Omega_{*}, k_{*}\right) \supset\left(\{0<|z|<1 / 2\}, k_{*}\right) \stackrel{\text { id }}{\rightarrow}\left(\{0<|z|<1 / 2\}, k_{\star}\right) \subset\left(\mathbb{C}_{\star}, k_{\star}\right)
$$

is an isometric equivalence and thus Lemma 2.8 gives the first assertion. The second assertion holds because $z \mapsto z^{-1}$ is quasihyperbolically 2-bi-Lipschitz, as per Fact 2.10. Alternatively, it is not hard to check that for $|z| \geq 2, \frac{1}{2}|z| \leq \delta^{*}(z) \leq$ $|z|=\delta_{\star}(z)$. 
We conclude this subsubsection with a technical result that we employ in our proof of Theorem 3.9. As above there are separate cases for finite versus infinite punctures.

3.13. Proposition. Suppose $\mathbb{D} \subset \Omega \subset \mathbb{C}$ with $1 \in \mathbb{C} \backslash \Omega$. Assume $\Omega_{*}:=\Omega \backslash\{0\}$ is hyperbolic and let $h_{*}:=h_{\Omega_{*}}, k_{*}:=k_{\Omega_{*}}$. For each $r \in\left(0, \frac{1}{2}\right],\left(\Delta_{*}, h_{*}\right)$ is $C_{1}$-roughly isometrically equivalent to $\left(\Delta_{*}, k_{*}\right)$, where $\Delta_{*}:=\mathrm{D}[0 ; r] \backslash\{0\}$ and $C_{1}:=\pi / \log 2$ $\leq 5$.

Next, suppose $1 \in \hat{\mathbb{C}} \backslash \Omega \subset \overline{\mathbb{D}}$. Assume $\Omega^{*}:=\Omega \backslash\{\infty\}$ is hyperbolic and let $h^{*}:=$ $h_{\Omega^{*}}, k^{*}:=k_{\Omega^{*}}$. For each $R \geq 4,\left(\Delta^{*}, h^{*}\right)$ is $(2,2 \pi)$-quasiisometrically equivalent to $\left(\Delta^{*}, k^{*}\right)$, where $\Delta^{*}:=\mathbb{C} \backslash \mathrm{D}(0 ; R)$.

Proof. First, suppose $\mathbb{D} \subset \Omega \subset \mathbb{C}$ with $1 \in \mathbb{C} \backslash \Omega$ and $\Omega_{*}=\Omega \backslash\{0\}$ hyperbolic. Fix $r \in\left(0, \frac{1}{2}\right]$ and let $\Delta_{*}:=\mathrm{D}[0 ; r] \backslash\{0\}$. As in Lemma 3.12,

$$
([0,+\infty),|\cdot|) \stackrel{\vartheta}{\rightarrow}\left(\Delta_{*}, k_{*}\right) \subset\left(\Omega_{*}, k_{*}\right), \quad \vartheta(s):=r e^{-s}
$$

is an isometric embedding and a $\pi$-rough isometric equivalence.

We show that the map

$$
\left(\Delta_{*}, h_{*}\right) \stackrel{\psi}{\rightarrow}([0,+\infty),|\cdot|) \quad \text { given by } \quad \psi(z):=\log \frac{\log (1 /|z|)}{\log (1 / r)}
$$

is a surjective rough isometric equivalence. The inclusions $\Delta_{*} \subset \mathbb{D}_{\star} \subset \Omega_{*} \subset \mathbb{C}_{01}$ tell us that

$$
\text { in } \mathbb{D}_{\star}, \quad h_{\star} \geq h_{*} \geq h_{01} \quad\left(\text { here } h_{\star}=h_{\mathbb{D}_{\star}} \text { and } h_{01}=h_{\mathbb{C}_{01}}\right) .
$$

Employing the estimates in Facts 2.13 we deduce that for all $0<|a| \leq|b| \leq \frac{1}{2}$ :

$$
h_{*}(a, b) \leq h_{\star}(a, b) \leq \log \frac{\log (1 /|a|)}{\log (1 /|b|)}+\frac{\pi}{\log 2}
$$

and

$$
h_{*}(a, b) \geq h_{01}(a, b) \geq \log \frac{\mathrm{k}+\log (1 /|a|)}{\mathrm{k}+\log (1 /|b|)} \geq \log \left(\frac{\log 2}{\mathrm{k}+\log } \frac{\log (1 /|a|)}{\log (1 /|b|)}\right) ;
$$

here (3.11) provides the last inequality. Thus for all $a, b \in \Delta_{*}$,

$$
h_{*}(a, b)-\frac{\pi}{\log 2} \leq|\psi(a)-\psi(b)| \leq h_{*}(a, b)+\log \left(1+\frac{\mathrm{k}}{\log 2}\right)
$$

and so $\psi$ is indeed a surjective $\left(1, C_{1}\right)$-QI equivalence.

It is now not difficult to confirm that the map

$$
\begin{gathered}
\left(\Omega_{*}, h_{*}\right) \supset\left(\Delta_{*}, h_{*}\right) \stackrel{\Phi:=\vartheta \circ \psi}{\longrightarrow}\left(\Delta_{*}, k_{*}\right) \subset\left(\Omega_{*}, k_{*}\right), \\
\Phi(z):=\vartheta(\psi(z))=r e^{-\psi(z)}=\frac{r \log (1 / r)}{\log (1 /|z|)}
\end{gathered}
$$

is a $C_{1}$-rough isometric equivalence.

Next, we examine an infinite puncture. Suppose $1 \in \hat{\mathbb{C}} \backslash \Omega \subset \overline{\mathbb{D}}$ with $\Omega^{*}=\Omega \backslash\{\infty\}$ hyperbolic. Fix $R \geq 4$ and let $\Delta^{*}:=\mathbb{C} \backslash \mathrm{D}(0 ; R)$. Here we cannot argue as in the second part of the proof of Lemma 3.12 because we do not know whether the origin lies in $\Omega$ or in $\mathbb{C} \backslash \Omega$. Also, unlike the first part above, where we had the three boundary points $0,1, \infty$, here we only know two boundary points. Nonetheless, 
both $\left(\Delta^{*}, h^{*}\right)$ and $\left(\Delta^{*}, k^{*}\right)$ are QI equivalent to the infinite ray $([0,+\infty),|\cdot|)$ as we now corroborate.

Put $R_{1}:=R+1, \Delta_{1}:=\hat{\mathbb{C}} \backslash \mathrm{D}\left(1 ; R_{1}\right)$, and $\Delta_{1}^{*}:=\Delta_{1} \backslash\{\infty\}$. One can appeal to Lemma 3.12 with $\Delta_{1}^{*}$, but a direct approach yields better estimates. It is not hard to check that for $|z-1| \geq 4$,

$$
\frac{1}{2}|z-1| \leq \delta^{*}(z) \leq|z-1| .
$$

Arguing as in the alternative proof for the second assertion in Lemma 3.12, we deduce that the map

$$
([0,+\infty),|\cdot|) \stackrel{\vartheta}{\rightarrow}\left(\Delta^{*}, k^{*}\right) \quad \text { given by } \vartheta(s):=1-R_{1} e^{s}
$$

satisfies

$$
s, t \in[0,+\infty) \Longrightarrow|s-t| \leq k^{*}(\vartheta(s), \vartheta(t)) \leq 2|s-t|
$$

with

$$
\vartheta([0,+\infty))=(-\infty,-R] \subset \Delta^{*} \subset N_{k^{*}}[(-\infty,-R] ; 2 \pi] .
$$

It follows that $\vartheta$ is a $(2,2 \pi)$-QI equivalence.

Pick any $\xi \in \hat{\mathbb{C}} \backslash \Omega$ with $|\xi-1|=\max _{\zeta \in \hat{\mathbb{C}} \backslash \Omega}|\zeta-1| 13$ so $|\xi| \leq 1,0<|\xi-1| \leq 2$, and $\hat{\mathbb{C}} \backslash \Omega \subset \mathrm{D}[1 ;|\xi-1|]$.

Let $\Delta^{\prime}:=T\left(\Delta_{1}^{*}\right), \Omega^{\prime}:=T\left(\Omega^{*}\right)$, and $h^{\prime}:=h_{\Omega^{\prime}}$ where $\left(\Omega^{*}, h^{*}\right) \stackrel{T}{\rightarrow}\left(\Omega^{\prime}, h^{\prime}\right)$ is the isometric equivalence given by

$$
T(z):=\frac{z-1}{\xi-1}
$$

Since $T(\mathrm{D}[1 ;|\xi-1|])=\overline{\mathbb{D}}$ and $1, \xi \in \hat{\mathbb{C}} \backslash \Omega \subset \mathrm{D}[1 ;|\xi-1|]$, we find that

$$
0,1 \in \hat{\mathbb{C}} \backslash \Omega^{\prime} \subset \overline{\mathbb{D}}, \quad \text { whence } \quad \mathbb{D}^{\star}:=\mathbb{C} \backslash \overline{\mathbb{D}} \subset \Omega^{\prime} \subset \mathbb{C}_{01}
$$

and therefore in $\mathbb{D}^{\star}, h^{\star}:=h_{\mathbb{D}^{\star}} \geq h^{\prime} \geq h_{01}$.

In particular, for all points $a^{\prime}, b^{\prime} \in \Delta^{\prime}$ (with $\left|b^{\prime}\right| \geq\left|a^{\prime}\right| \geq R_{2}:=R_{1} /|\xi-1|$ ) we have

$$
h^{\prime}\left(a^{\prime}, b^{\prime}\right) \leq h^{\star}\left(a^{\prime}, b^{\prime}\right) \leq \log \frac{\log \left|b^{\prime}\right|}{\log \left|a^{\prime}\right|}+\frac{\pi}{\log 2}
$$

and

$$
h^{\prime}\left(a^{\prime}, b^{\prime}\right) \geq h_{01}\left(a^{\prime}, b^{\prime}\right) \geq \log \frac{\mathrm{k}+\log \left|b^{\prime}\right|}{\mathrm{k}+\log \left|a^{\prime}\right|} \geq \log \left(\frac{L}{\mathrm{k}+L} \frac{\log \left|b^{\prime}\right|}{\log \left|a^{\prime}\right|}\right) ;
$$

here $L:=\log R_{2} \geq \log (5 / 2)>0.9$, (3.11) provides the last inequality, and we have used Fact 2.13(c). Thus for all $a^{\prime}, b^{\prime} \in \Delta^{\prime}$,

$$
h^{\prime}\left(a^{\prime}, b^{\prime}\right)-\frac{\pi}{\log 2} \leq\left|\psi\left(a^{\prime}\right)-\psi\left(b^{\prime}\right)\right| \leq h^{\prime}\left(a^{\prime}, b^{\prime}\right)+\log \left(1+\frac{\mathrm{k}}{L}\right)
$$

${ }^{13}$ Note that $|\xi-1| \geq \frac{1}{2} \operatorname{diam}(\hat{\mathbb{C}} \backslash \Omega)>0$ because $\Omega^{*}$ is hyperbolic. 
where

$$
\left(\Delta^{\prime}, h^{\prime}\right) \stackrel{\psi}{\rightarrow}([0,+\infty),|\cdot|) \quad \text { is given by } \quad \psi(w):=\log \frac{\log |w|}{L}
$$

and $\psi$ is a surjective $\left(1, C_{1}\right)$-QI equivalence.

Next, we employ Example 2.1 to demonstrate that $\left(\Delta^{*}, h^{*}\right)$ is roughly isometrically equivalent to $\left(\Delta_{1}^{*}, h^{*}\right)$ (and hence to $\left(\Delta^{\prime}, h^{\prime}\right)$ ). We claim that for each $z \in \Delta^{*}$ there is a $z_{1} \in \Delta_{1}^{*}$ with $h^{*}\left(z, z_{1}\right) \leq \frac{1}{2}$.

Let $z_{0} \in \Delta^{*}$. If $z_{0} \in \Delta_{1}^{*}$, put $z_{1}:=z_{0}$; assume $z_{0} \notin \Delta_{1}^{*}$. Let $z_{1}$ be the radial projection, from the origin, of $z_{0}$ onto $\partial \Delta_{1}^{*}$. Note that $\left|z_{0}-z_{1}\right| \leq 2$. Also: $\left[z_{0}, z_{1}\right] \subset\left[\left(R /\left|z_{0}\right|\right) z_{0}, z_{1}\right]$ and for each $z \in\left[\left(R /\left|z_{0}\right|\right) z_{0}, z_{1}\right],|z| \geq R \geq 4$; so

$$
\lambda^{\star}(z)=\frac{1}{|z| \log |z|} \leq \frac{1}{|z|} \leq \frac{1}{R} \leq \frac{1}{4} .
$$

In $\mathbb{D}^{\star} \subset \Omega^{*}, \lambda^{\star} \geq \lambda^{*}$, and thus

$$
h^{*}\left(z_{0}, z_{1}\right) \leq h^{\star}\left(z_{0}, z_{1}\right)=\int_{\left[z_{0}, z_{1}\right]} \lambda^{\star} d s \leq \max _{\left[z_{0}, z_{1}\right]} \lambda^{\star} \cdot\left|z_{0}-z_{1}\right| \leq \frac{1}{2} .
$$

From Example 2.1, the map $\left(\Delta^{*}, h^{*}\right) \stackrel{f}{\rightarrow}\left(\Delta_{1}^{*}, h^{*}\right), f(z):=z_{1}$ (with $z_{1}$ as given in the above claim), is a 1-rough isometric equivalence.

Finally, we now have

$$
\left(\Delta^{*}, h^{*}\right) \stackrel{f}{\rightarrow}\left(\Delta_{1}^{*}, h^{*}\right) \stackrel{T}{\rightarrow}\left(\Delta^{\prime}, h^{\prime}\right) \stackrel{\psi}{\rightarrow}([0,+\infty),|\cdot|) \stackrel{\vartheta}{\rightarrow}\left(\Delta^{*}, k^{*}\right)
$$

and a careful review of our estimates reveals that

$$
\Psi:=\psi \circ T \circ f, \quad \Psi(z)=\log \frac{\log \left|w_{1}\right|}{\log R_{2}} \text { with } w_{1}:=T\left(z_{1}\right),
$$

is a rough isometric equivalence. Moreover, $\Phi:=\vartheta \circ \Psi$ is $(2,6)$-QI with

$$
\Phi\left(\Delta^{*}\right)=\vartheta([0,+\infty))=(-\infty,-R] \subset \Delta^{*} \subset N_{k^{*}}[(-\infty,-R] ; 2 \pi],
$$

and thus $\Phi$ provides the asserted QI equivalence between $\left(\Delta^{*}, h^{*}\right)$ and $\left(\Delta^{*}, k^{*}\right)$.

3.14. Corollary. Let $\Pi \subset \Omega \subset \hat{\mathbb{C}}$ be as described in $₫$ 3.C.1. Then for each $p \in \Pi$ there is an $(L, C)$-quasiisometric equivalence

$$
\left(\Omega_{\Pi}, h_{\Pi}\right) \supset\left(\Delta_{p}, h_{p}\right) \stackrel{\Phi_{p}}{\longrightarrow}\left(\Delta_{p}, k_{p}\right) \subset\left(\Omega_{\Pi}, k_{\Pi}\right)
$$

where $h_{p}$ and $k_{p}$ denote the distances $h_{\Pi}$ and $k_{\Pi}$ restricted to $\Delta_{p}$ (respectively). We can take $(L, C)=(1, \pi / \log 2)$ unless $p=\infty$ in which case $(L, C)=(2,2 \pi)$.

Proof. First, assume $p \in \Pi \backslash\{\infty\}$. Let $\Omega_{p}:=\Omega_{\Pi} \cup\{p\}$ and pick a point $\xi \in$ $\partial \Omega_{p}$ nearest to $p$. Put $T(z):=(z-p) /(\xi-p)$. Then $\Omega^{\prime}:=T\left(\Omega_{p}\right)$ satisfies the hypotheses of the first part of Proposition 3.13 . Also, $r_{p}^{\prime}:=r_{p} /|\xi-p| \leq \frac{1}{2}$, so there is a $\pi / \log 2$-rough isometric equivalence $\Phi$ from $\left(\Delta_{*}^{\prime}, h^{\prime}\right)$ to $\left(\Delta_{*}^{\prime}, k^{\prime}\right)$, where $\Delta_{*}^{\prime}=\mathrm{D}\left(0 ; r_{p}^{\prime}\right) \backslash\{0\}=T\left(\Delta_{p}\right) \subset \Omega^{\prime}$ (and $h^{\prime}, k^{\prime}$ are respectively the hyperbolic, quasihyperbolic distances in $\Omega_{*}^{\prime}=\Omega^{\prime} \backslash\{0\}=T\left(\Omega_{\Pi}\right)$ ). It now follows that

$$
\Phi_{p}:=T^{-1} \circ \Phi \circ T, \quad \Phi_{p}(z)=p+r_{p} \frac{\xi-p}{|\xi-p|} \frac{\log \left(r_{p} /|\xi-p|\right)}{\log (|z-p| /|\xi-p|)},
$$

is a $\pi / \log 2$-rough isometric equivalence from $\left(\Delta_{p}, h_{p}\right)$ to $\left(\Delta_{p}, k_{p}\right)$. 
Next, assume $p=\infty \in \Pi$. Put $\Omega_{\infty}:=\Omega_{\Pi} \cup\{\infty\}$. Pick a point $\xi \in \hat{\mathbb{C}} \backslash \Omega_{\infty}$ with $|\xi|=\max _{\zeta \in \hat{\mathbb{C}} \backslash \Omega_{\infty}}|\zeta|$. Let $T(z):=z / \xi$. Then $\Omega^{\prime}:=T\left(\Omega_{\infty}\right)$ satisfies the hypotheses of the second part of Proposition [3.13. Also, $R^{\prime}:=r_{\infty} /|\xi| \geq 4$, so there is a $(2,2 \pi)$-quasiisometric equivalence $\Phi$ from $\left(\Delta^{\prime *}, h^{\prime}\right)$ to $\left(\Delta^{\prime *}, k^{\prime}\right)$, where $\Delta^{\prime *}=\mathbb{C} \backslash \mathrm{D}\left(0 ; R^{\prime}\right)=T\left(\Delta_{\infty}^{\star}\right) \subset \Omega^{\prime}$ (and $h^{\prime}, k^{\prime}$ are respectively the hyperbolic, quasihyperbolic distances in $\Omega^{\prime *}=\Omega^{\prime} \backslash\{\infty\}=T\left(\Omega_{\Pi}\right)$ ). It now follows that

$$
\Phi_{\infty}:=T^{-1} \circ \Phi \circ T, \quad \Phi_{\infty}(z)=\xi \Phi(z / \xi),
$$

is a $(2,2 \pi)$-quasiisometric equivalence from $\left(\Delta_{\infty}^{\star}, h_{\infty}\right)$ to $\left(\Delta_{\infty}^{\star}, k_{\infty}\right)$.

3.C.3. Proof of Theorem 3.9. The reader is encouraged to review 3.C.1, In particular, note that $\hat{\mathbb{C}} \backslash \Omega_{\Delta}$ uniformly perfect tells us that each isolated point of $\hat{\partial} \Omega$ is a limit point of $\Pi$.

Define $\left(\Omega_{\Pi}, h_{\Pi}\right) \stackrel{\Phi}{\rightarrow}\left(\Omega_{\Pi}, k_{\Pi}\right)$ by

$$
\Phi(z):= \begin{cases}\Phi_{p}(z) & \text { if } z \in \Delta_{p}^{\star} \\ z & \text { if } z \in \Omega_{\Delta}\end{cases}
$$

here $\left(\Delta_{p}^{\star}, h_{\Pi}\right) \stackrel{\Phi_{p}}{\longrightarrow}\left(\Delta_{p}^{\star}, k_{\Pi}\right)$ are the $(2,2 \pi)$-QI equivalences given by Corollary 3.14 Below we demonstrate that the identity map $\left(\Omega_{\Delta}, h_{\Pi}\right) \rightarrow\left(\Omega_{\Delta}, k_{\Pi}\right)$ is $K$-bi-Lipschitz with $K=K(M)$. An elementary, albeit tedious, argument then reveals that $\Phi$ is $2 \pi$-roughly surjective and

$$
\forall a, b \in \Omega_{\Pi}, \quad \frac{1}{2} h_{\Pi}(a, b)-6 \pi \leq k_{\Pi}(\Phi(a), \Phi(b)) \leq K h(a, b)+6 \pi ;
$$

so $\Phi$ is a $(K, 6 \pi)$-quasiisometric equivalence; we assume $K \geq 2$.

To begin, we demonstrate that each annulus $A \in \mathcal{A}_{\Pi}$ (so, $A \subset \Omega_{\Pi}$ and $c(A) \in$ $\left.\mathbb{C} \backslash \Omega_{\Pi}\right)$ with $\mathrm{S}^{1}(A) \cap \Delta=\emptyset$ has modulus $\bmod (A) \leq 2 M+\log 4$. Let $A:=$ $\{z \in \mathbb{C}|r<| z-c \mid<R\}$ be such an annulus. Assume $R / r>4$; so, $\frac{3}{2} r<\sqrt{r R}<$ $\frac{1}{2} R$. Set

$$
\Pi_{\text {in }}:=\Pi \cap A_{\text {in }}, \quad \Pi_{\text {out }}:=\Pi \cap\left(A_{\text {out }} \cup\{\infty\}\right), \quad \Pi_{\text {out }}^{\star}:=\Pi_{\text {out }} \backslash\{\infty\} .
$$

Define $A^{\prime}:=\left\{z \in \mathbb{C}\left|r^{\prime}<\right| z-c \mid<R^{\prime}\right\}$ where

$$
r^{\prime}:= \begin{cases}r & \text { if } \Pi_{\text {in }}=\emptyset, \text { or, } \Pi_{\text {in }}=\{c\} \text { and } A_{\text {in }} \cap(\mathbb{C} \backslash \Omega) \neq \emptyset, \\ \frac{3}{2} r & \text { if } \left.\Pi_{\text {in }} \backslash\{c\} \neq \emptyset \text { (i.e., } c \notin \Pi_{\text {in }} \neq \emptyset \text { or }\{c\} \subsetneq \Pi_{\text {in }}\right), \\ \sqrt{r R} & \text { if } \Pi_{\text {in }}=\{c\} \text { and } A_{\text {in }} \cap(\mathbb{C} \backslash \Omega)=\emptyset .\end{cases}
$$

and

$$
R^{\prime}:= \begin{cases}R & \text { if } \Pi_{\text {out }}=\emptyset, \text { or, } \Pi_{\text {out }}=\{\infty\} \text { and } A \cap \Delta_{\infty}=\emptyset, \\ \frac{1}{2} R & \text { if } \left.\Pi_{\text {out }}^{*} \neq \emptyset \text { (i.e., }\{\infty\} \neq \Pi_{\text {out }} \neq \emptyset\right), \\ \sqrt{r R} & \text { otherwise (i.e., } \Pi_{\text {out }}=\{\infty\} \text { and } A \cap \Delta_{\infty} \neq \emptyset \text { ). }\end{cases}
$$

We show below that when $R^{\prime}=\sqrt{r R}, A_{\text {out }} \cap\left(\mathbb{C} \backslash \Omega_{\Pi}\right)=\emptyset$. Since $\Omega_{\Pi}$ is hyperbolic, $A_{\text {out }} \cap\left(\mathbb{C} \backslash \Omega_{\Pi}\right)=\emptyset=A_{\text {in }} \cap(\mathbb{C} \backslash \Omega)$ cannot both hold (as these would imply $\left.\hat{\mathbb{C}} \backslash \Omega_{\Pi}=\{c, \infty\}\right)$. Thus $r \leq r^{\prime}<R^{\prime} \leq R, A^{\prime} \Subset A$ is a concentric subannulus of $A$, and it is not difficult to check that $\log (R / r) \leq 2 \log \left(R^{\prime} / r^{\prime}\right)+\log 4$. We claim that $A^{\prime} \cap \Delta=\emptyset$. Thus $A^{\prime} \subset \Omega_{\Delta}$; since $\hat{\mathbb{C}} \backslash \Omega_{\Delta}$ is $M$-uniformly perfect, $\bmod \left(A^{\prime}\right) \leq M$ and $\bmod (A) \leq 2 M+\log 4$ as asserted. 
Now we check that $A^{\prime} \cap \Delta=\emptyset$. Employing either (3.10a) if $c \in \partial \Omega$ or (3.10b) if $c \in \Pi^{\star}$ we find that

$$
\forall p \in \Pi^{\star} \backslash\{c\}, \quad 2 r_{p} \leq|p-c| .
$$

First we show that for all $q \in \Pi_{\mathrm{in}}, \Delta_{q} \subset \mathrm{D}\left[c ; r^{\prime}\right]$. Assume $\Pi_{\mathrm{in}} \neq \emptyset$. If $c \notin \Pi_{\mathrm{in}}$, then (3.15) provides the estimate $r_{q} \leq \frac{1}{2} r$ for $q \in \Pi_{\text {in }}$, so $\Delta_{q} \subset \mathrm{D}\left[c ; \frac{3}{2} r\right]$ as asserted. A similar argument works for the case $\{c\} \subsetneq \Pi_{\mathrm{in}}$. Assume $\{c\}=\Pi_{\mathrm{in}}$. Suppose $A_{\text {in }} \cap(\mathbb{C} \backslash \Omega) \neq \emptyset$, and let $\zeta$ be a point in this set. By (3.10a), $2 r_{c} \leq|c-\zeta| \leq r$, so $\Delta_{c} \subset \mathrm{D}[c ; r]$ as asserted. When $A_{\text {in }} \cap(\mathbb{C} \backslash \Omega)=\emptyset$, we have no estimates for $r_{c}$, but $\mathrm{S}^{1}(A) \cap \Delta=\emptyset$ means that $\Delta_{c} \subset \mathrm{D}[c ; \sqrt{r R}]$.

Next we show that for all $q \in \Pi_{\text {out }}, \Delta_{q} \cap \mathrm{D}\left(c ; R^{\prime}\right)=\emptyset$. Assume $\Pi_{\text {out }} \neq \emptyset$. Suppose $q \in \Pi_{\text {out }}^{\star}$. If $r_{q} \leq \frac{1}{2} R$, then $|q-c|-r_{q} \geq \frac{1}{2} R$, and if $r_{q} \geq \frac{1}{2} R$, then by (3.15), $|q-c|-r_{q} \geq r_{q} \geq \frac{1}{2} R$; thus, in both cases $\Delta_{q} \cap \mathrm{D}\left(c ; \frac{1}{2} R\right)=\emptyset$. Also, if $\infty \in \Pi$, then $|c|+R \leq|c|+|q-c| \leq \frac{3}{4} r_{\infty}$, so here $\Delta_{\infty} \cap \mathrm{D}(c ; R)=\emptyset$.

It remains to examine the case $\Pi_{\text {out }}=\{\infty\}$ and $A \cap \Delta_{\infty} \neq \emptyset$. Here we have no estimates for $r_{\infty}$, but $\mathrm{S}^{1}(A) \cap \Delta=\emptyset$ means that $\Delta_{\infty} \cap \mathrm{D}[c ; \sqrt{r R}]=\emptyset$. Also, in this setting we must have $A_{\text {out }} \cap\left(\mathbb{C} \backslash \Omega_{\Pi}\right)=\emptyset$. (If there were a point $\zeta \in A_{\text {out }} \cap\left(\mathbb{C} \backslash \Omega_{\Pi}\right)$, then by (3.10c), $R \leq|\zeta-c| \leq|\zeta|+|c| \leq \frac{1}{2} r_{\infty}$, which would give $A \subset \mathrm{D}\left[0 ; \frac{3}{4} r_{\infty}\right]$ contradicting $A \cap \Delta_{\infty} \neq \emptyset$.)

We can use the above to verify that $\mathrm{bp}_{\Pi} \leq M+2$ in $\Omega_{\Delta}$ which tells us that $\lambda_{\Pi} d s$ and $\delta_{\Pi}^{-1} d s$ are $(\mathrm{k}+M+2)$-bi-Lipschitz equivalent in $\Omega_{\Delta}$. However, if $\gamma: a \curvearrowright b$ is either a hyperbolic or a quasihyperbolic geodesic in $\Omega_{\Pi}$ with endpoints $a, b \in \Omega_{\Delta}$, then $\gamma$ may leave $\Omega_{\Delta}$. Nonetheless, the ABC property (see 2.C.4) ensures that $\gamma$ cannot enter too deep into $\Delta \backslash \Pi=\Omega_{\Pi} \backslash \Omega_{\Delta}$. In particular, if $\gamma$ enters some $\Delta_{p}$, then by Fact $2.16(\mathrm{a}),|\gamma| \cap \mathrm{D}\left[p ; e^{-6 \mathrm{k}} r_{p}\right]=\emptyset$ when $p \neq \infty$ and $|\gamma| \subset \mathrm{D}\left(0 ; e^{6 \mathrm{k}} r_{\infty}\right)$ when $p=\infty$.

Our final task is to corroborate that $\mathrm{bp}_{\Pi} \lesssim 1$ in $\Omega_{\tilde{\Delta}}:=\Omega \backslash \tilde{\Delta}$ where $\tilde{\Delta}:=\bigcup_{p \in \Pi} \tilde{\Delta}_{p}$ and

$$
\tilde{\Delta}_{p}:= \begin{cases}\mathrm{D}\left[p ; e^{-6 \mathrm{k}} r_{p}\right] & \text { if } p \in \Pi^{\star}, \\ \hat{\mathbb{C}} \backslash \mathrm{D}\left(0 ; e^{6 \mathrm{k}} r_{\infty}\right) & \text { if } p=\infty \in \Pi .\end{cases}
$$

To this end, let $a \in \Omega_{\tilde{\Delta}}$ and pick $c \in \partial \Omega_{\Pi}$ with

$A:=\mathrm{BP}_{\Pi}(a)=\mathrm{A}(c, d ; m)=\left\{d e^{-m}<|z-c|<d e^{m}\right\}=\{r<|z-c|<R\} \in \mathcal{A}_{\Pi}$ where $d:=\delta_{\Pi}(a)=|a-c|$ and $\operatorname{bp}_{\Pi}(a)=m=\frac{1}{2} \log (R / r)$. Recall (3.15) and the notation $\Pi_{\text {in }}, \Pi_{\text {out }}$. We assume $S^{1}(A) \cap \Delta \neq \emptyset$, so $\Upsilon:=\left\{p \in \Pi \mid \mathrm{S}^{1}(A) \cap \Delta_{p} \neq \emptyset\right\} \neq$ $\emptyset$.

We consider several cases. If $p \in \Upsilon \cap \Pi_{\text {in }} \backslash\{c\}$, then by (3.15)

$$
|p-c| \leq r \quad \text { and } \quad d \leq|p-c|+r_{p} \leq \frac{3}{2}|p-c|
$$

so

$$
\sqrt{R / r}=\frac{d}{r} \leq \frac{3}{2} \quad \text { whence } \quad \frac{R}{r} \leq \frac{9}{4}<3
$$

If $p \in \Upsilon \cap \Pi_{\text {out }}^{\star}$, then again by (3.15)

$$
|p-c| \geq R \quad \text { and } \quad d+r_{p} \geq|p-c|, \quad \text { so } \quad d \geq \frac{1}{2}|p-c|
$$


and therefore

$$
\sqrt{R / r}=\frac{R}{d} \leq 2 \text { and } \frac{R}{r} \leq 4
$$

Thus in these two easy cases we have $\operatorname{bp}_{\Pi}(a)=\frac{1}{2} \bmod (A) \leq \log 2$.

It remains to deal with the case $\Upsilon \cap\{c, \infty\} \neq \emptyset$; here $\mathrm{S}^{1}(A) \cap \Delta_{q}=\emptyset$ for all $q \in \Pi^{\star} \backslash\{c\}$. Roughly speaking, we exhibit a concentric subannulus $A^{\prime} \subset A$ with $\bmod A^{\prime} \simeq \bmod A$ and $\mathrm{S}^{1}\left(A^{\prime}\right) \cap \Delta=\emptyset$. It then follows from earlier work that $\bmod A \lesssim M$.

Suppose $c \in \Upsilon$. Then $\Upsilon=\{c\}, r_{c} \geq d$, and $R \geq 2 r_{c}$ (because $c$ is an isolated point of $\partial \Omega_{\Pi}$ nearest to $a$, so $\left.\partial_{\text {out }} A \cap \partial \Omega_{\Pi} \neq \emptyset\right)$. If $R \leq 2 r_{c} e^{m / 2}$, then as $a \notin \tilde{\Delta}_{p}$,

$$
2 r_{c} \geq R e^{-m / 2}=d e^{m / 2}=|a-c| e^{m / 2} \geq e^{-6 \mathrm{k}} r_{c} e^{m / 2}
$$

whence

$$
\mathrm{bp}(a)=m \leq 12 \mathrm{k}+\log 4 .
$$

Assume $R \geq 2 r_{c} e^{m / 2}$. We claim that $r^{\prime}:=\left(2 r_{c}\right)^{2} / R \in[r, d]$. Therefore

$$
A^{\prime}:=\left\{z \in \mathbb{C}\left|r^{\prime}<\right| z-c \mid<R\right\} \Subset A \text { is a concentric subannulus of } A
$$

with $\mathrm{S}^{1}\left(A^{\prime}\right)=\mathrm{S}^{1}\left(c ; 2 r_{c}\right)$. From (3.10), $\mathrm{S}^{1}\left(A^{\prime}\right) \cap \Delta=\emptyset$, so by earlier work,

$$
\mathrm{bp}(a)=\frac{1}{2} \bmod (A)=\log \frac{R}{d} \leq \log \frac{R}{r^{\prime}}=\bmod \left(A^{\prime}\right) \leq 2 M+\log 4 .
$$

To check the claim, note that

$$
r=\frac{d^{2}}{R} \leq \frac{r_{c}^{2}}{R} \leq r^{\prime}=\frac{\left(2 r_{c}\right)^{2}}{R} \leq \frac{\left(R e^{-m / 2}\right)^{2}}{R}=R e^{-m}=d .
$$

Suppose $\infty \in \Upsilon$. Here $\Upsilon=\{\infty\}, \frac{3}{4} r_{\infty} \leq d \leq C r_{\infty}$ where $C:=e^{6 \mathrm{k}}+\frac{1}{4}$, and, $r \leq \frac{1}{2} r_{\infty}$ (because $\partial_{\text {in }} A \cap \partial \Omega_{\Pi} \neq \emptyset$ ). If $r \geq \frac{1}{2} r_{\infty} e^{-m / 2}$, then

$$
\frac{1}{2} r_{\infty} \leq r e^{m / 2}=d e^{-m / 2} \leq C r_{\infty} e^{-m / 2}
$$

whence

$$
\mathrm{bp}(a)=m \leq 12 \mathrm{k}+4 .
$$

Assume $r \leq \frac{1}{2} r_{\infty} e^{-m / 2}$. We claim that $R^{\prime}:=\left(\frac{1}{2} r_{\infty}\right)^{2} / r \in\left[d, \frac{4}{9} R\right]$. Therefore

$$
A^{\prime}:=\left\{z \in \mathbb{C}|r<| z-c \mid<R^{\prime}\right\} \subset A \text { is a concentric subannulus of } A
$$

with $\mathrm{S}^{1}\left(A^{\prime}\right)=\mathrm{S}^{1}\left(c ; \frac{1}{2} r_{\infty}\right)$. From (3.10c), $\mathrm{S}^{1}\left(A^{\prime}\right) \cap \Delta=\emptyset$, so by earlier work,

$$
\mathrm{bp}(a)=\frac{1}{2} \bmod (A)=\log \frac{d}{r} \leq \log \frac{R^{\prime}}{r}=\bmod \left(A^{\prime}\right) \leq 2 M+\log 4 .
$$

To check the claim, note that

$$
d=r e^{m}=\frac{\left(r e^{m / 2}\right)^{2}}{r} \leq \frac{\left(\frac{1}{2} r_{\infty}\right)^{2}}{r}=R^{\prime} \leq \frac{\left(\frac{2}{3} d\right)^{2}}{r}=\frac{4 r R}{9 r}=\frac{4}{9} R .
$$


Having established that $\mathrm{bp}_{\Pi} \leq M^{\prime}:=\max \{2 M+\log 4,12 \mathrm{k}+4\}$ in $\Omega_{\tilde{\Delta}}$, we now know that $\lambda_{\Pi} d s$ and $\delta_{\Pi}^{-1} d s$ are $K$-bi-Lipschitz equivalent in $\Omega_{\tilde{\Delta}}$ with

$$
K:=K(M)=\mathrm{k}+M^{\prime} .
$$

As explained above, any hyperbolic or quasihyperbolic geodesic in $\Omega_{\Pi}$ with endpoints in $\Omega_{\Delta}$ lies in $\Omega_{\tilde{\Delta}}$. Therefore the identity $\operatorname{map}\left(\Omega_{\Pi}, h_{\Pi}\right) \rightarrow\left(\Omega_{\Pi}, k_{\Pi}\right)$ is $K$-biLipschitz.

3.D. Proof of Theorem D. Here we concoct plane domains $\Omega$ that satisfy the assertions of Theorem D. We require the following technical fact about quasisymmetric maps; this must be folklore, but we do not know a reference.

3.16. Lemma. Let $\left(a_{n}\right)_{0}^{\infty}$ be a strictly increasing sequence in $\mathbb{R} \backslash\{\infty\}$ with $a_{n+1} / a_{n}$ $\rightarrow+\infty$ as $n \rightarrow+\infty$. Put $A:=\left\{a_{n} \mid n \geq 0\right\} \cup\{\infty\} \subset \hat{\mathbb{R}}$. Then every QS homeomorphism $f: A \rightarrow A$ is "eventually the identity"; i.e., there is an $N$ such that for all $n \geq N, f\left(a_{n}\right)=a_{n} 14$

Proof. To start, note that as $f$ is a homeomorphism, it is a bijection and $f(\infty)=\infty$ (as $\infty$ is the only non-isolated point of $A$ ).

We exhibit $p, q$ such that for all $n \geq 1, f\left(a_{p+n}\right)=a_{q+n}$. Then $f$ maps $\left\{a_{0}, a_{1}, \ldots, a_{p}\right\}$ bijectively onto $\left\{a_{0}, a_{1}, \ldots, a_{q}\right\}$. Hence $p=q$ and our claim is established.

We need only produce a $p$ such that for all $n \geq p, f\left(a_{n}\right)<f\left(a_{n+1}\right)$ with $f\left(a_{n}\right)$ and $f\left(a_{n+1}\right)$ adjacent (meaning that if $f\left(a_{n}\right)=a_{m}$, then $\left.f\left(a_{n+1}\right)=a_{m+1}\right)$. Indeed, given such a $p$ we simply let $q$ be the unique integer with $a_{q}=f\left(a_{p}\right)$.

Below we use the quasisymmetry of $f$ to verify that there is an $N$ such that for all $n \geq N, f\left(a_{n}\right)<f\left(a_{n+1}\right)$. Let $M$ be the unique integer with $a_{M}=f\left(a_{N}\right)$. Thus for all $n \geq N, a_{M} \leq f\left(a_{n}\right)<f\left(a_{n+1}\right)$.

We claim that there are a finite number of $m \geq M$ such that $a_{m} \notin f\left(\left\{a_{n} \mid n \geq N\right\}\right)$. Indeed, if $m$ is such, then $a_{m} \in f\left(\left\{a_{0}, a_{1}, \ldots, a_{N-1}\right\}\right)$, so, there are at most $N$ such $m$. Let $\ell$ be the largest of all these $m$.

Thus there is a $p>N$ such that $a_{M} \leq f\left(a_{p-1}\right)<a_{\ell}<f\left(a_{p}\right)$, but for all $n \geq p$, $f\left(a_{n}\right)$ and $f\left(a_{n+1}\right)$ are adjacent. This is the sought after $p$. Since

To produce $N$, assume $f$ is $\eta$-QS. Pick $\tau \in(0,1)$ so that $t \in(0, \tau) \Longrightarrow \eta(t)<\frac{1}{2}$.

$$
\frac{\chi\left(a_{n+1}, \infty\right)}{\chi\left(a_{n}, \infty\right)} \leq 2 \frac{1+a_{n}}{1+a_{n+1}} \rightarrow 0
$$

there exists an $N$ such that for all $n \geq N$,

$$
t:=\frac{\chi\left(a_{n+1}, \infty\right)}{\chi\left(a_{n}, \infty\right)}<\tau
$$

Then for such $n$,

$$
\frac{\chi\left(f a_{n+1}, f \infty\right)}{\chi\left(f a_{n}, f \infty\right)} \leq \eta(t)<\frac{1}{2}
$$

${ }^{14}$ Here we use the chordal distance from $\hat{\mathbb{R}}:=\mathbb{R} \cup\{\infty\}$. 
whence

$$
\frac{4}{1+f\left(a_{n+1}\right)} \leq 2 \chi\left(f a_{n+1}, \infty\right) \leq \chi\left(f a_{n}, \infty\right) \leq \frac{4}{1+f\left(a_{n}\right)}
$$

and therefore $f\left(a_{n}\right)<f\left(a_{n+1}\right)$ as asserted.

3.17. Proof of Theorem D. Let $\left(a_{n}\right)_{1}^{\infty}$ be a strictly decreasing sequence in $(-\infty, 0)$ with $a_{n+1} / a_{n} \rightarrow+\infty$ as $n \rightarrow+\infty$. Put $a_{0}:=0, A:=\left\{a_{n} \mid n \geq 0\right\}$, and $\Omega:=\mathbb{C} \backslash A$. We verify that the assertions of Theorem $\mathrm{D}$ hold for $\Omega 15$

It is straightforward to check that $\Omega$ is a uniform domain; Her84, Lemma 8.4] provides a convenient criterion here.

We employ BHK01, Lemma 3.14] and its hyperbolic counterpart Proposition $3.4(\mathrm{f}),(\mathrm{g})$. To this end, note that the point $o:=1 \in \Omega$ has the property that $\sigma(1)=\operatorname{dist}_{\sigma}(1, \hat{\partial} \Omega)=\max _{\Omega} \sigma=\pi / 2$. Also, $[1,+\infty) \subset \mathbb{R} \subset \hat{\mathbb{C}}$ is both a hyperbolic and a quasihyperbolic geodesic ray in $\Omega$ from 1 to the boundary point at infinity.

Let $h_{\varepsilon}=h_{\varepsilon, o}$ and $k_{\varepsilon}=k_{\varepsilon, o}$ denote the standard visual distances on $\partial_{G}(\Omega, h)$ and $\partial_{G}(\Omega, k)$ repectively; as in $\$ 2 . \mathrm{D}$, the visual parameter $\varepsilon \in\left(0, \varepsilon_{0}\right]$ and $o=1 \in \Omega$ is the fixed base point.

Thanks to [BHK01, Theorem 3.6] and Theorem 3.3, we know that both $\partial_{G}(\Omega, k)$ and $\partial_{G}(\Omega, h)$ are naturally equivalent to $\hat{\partial} \Omega=\partial \Omega \cup\{\infty\}=A \cup\{\infty\}$. We use $a_{n}$ to denote any of: $a_{n} \in A \subset \partial \Omega$, the corresponding point in $\partial_{G}(\Omega, k)$, or the corresponding point in $\partial_{G}(\Omega, h)$. We also let $\zeta$ denote the boundary point at infinity in $\hat{\partial} \Omega$ and the corresponding points in $\partial_{G}(\Omega, k)$ and $\partial_{G}(\Omega, h)$.

Given a boundary point $\xi=a_{n}$, we easily check that the associated point $x \in$ $[o, \zeta)=[1, \infty)$ given by BHK01, Lemma 3.14] or Proposition 3.4(f) is $x=x_{n}:=$ $-a_{n}$. Employing Proposition 3.4 (g) (or its quasihyperbolic counterpart in [BHK01]) we deduce that for each distance function $d \in\{h, k\}$,

$$
C^{-1} e^{-\varepsilon d\left(x_{n}, 1\right)} \leq d_{\varepsilon}\left(a_{n}, \zeta\right) \leq C e^{-\varepsilon d\left(x_{n}, 1\right)},
$$

where $C=C(\varepsilon)$. It follows that

$$
\frac{k_{\varepsilon}\left(a_{n+1}, \zeta\right)}{k_{\varepsilon}\left(a_{n}, \zeta\right)} \leq C^{2} e^{-\varepsilon k\left(x_{n}, x_{n+1}\right)}
$$

and that

$$
\frac{h_{\varepsilon}\left(a_{n+1}, \zeta\right)}{h_{\varepsilon}\left(a_{n}, \zeta\right)} \geq C^{-2} e^{-\varepsilon h\left(x_{n}, x_{n+1}\right)}
$$

Note that

$$
\forall n \geq 1, \quad k\left(x_{n}, x_{n+1}\right)=\log \frac{x_{n+1}}{x_{n}}
$$

and, arguing as in Example 2.14,

$$
\forall n \geq N, \quad h\left(x_{n}, x_{n+1}\right) \leq 4+\pi \log \left(\frac{1}{2} \log \frac{x_{n+1}}{x_{n}}\right)
$$

where $N$ is chosen so that $n \geq N \Longrightarrow \log \left(x_{n+1} / x_{n}\right)>2$.

\footnotetext{
${ }^{15}$ The reader can check that there is no choice of $r_{p}>0$ such that the hypotheses in 3 .C.1 hold for $\Pi=A$ with $\Omega=\mathbb{C}$; the uniform perfectness of $\hat{\mathbb{C}} \backslash \Omega_{\Delta}$ always fails.
} 
Now suppose there were a power quasisymmetry $\partial_{G}(\Omega, k) \stackrel{f}{\rightarrow} \partial_{G}(\Omega, h)$; say, $f$ is $\eta$-QS with $\eta(t):=H\left(t^{\alpha} \vee t^{1 / \alpha}\right)$ for some constants $H>0$ and $\alpha \in(0,1]$. Then $f$ would induce a quasisymmetry of $A \cup\{\zeta\}$ onto itself, and hence by Lemma 3.16 we would know that $f(\zeta)=\zeta$ and $f\left(a_{n}\right)=a_{n}$ for all $n \geq N$.

It would then follow that: for all $n \geq N$,

$$
C^{-2} e^{-\varepsilon h\left(x_{n}, x_{n+1}\right)} \leq \frac{h_{\varepsilon}\left(a_{n+1}, \zeta\right)}{h_{\varepsilon}\left(a_{n}, \zeta\right)} \leq \eta\left(\frac{k_{\varepsilon}\left(a_{n+1}, \zeta\right)}{k_{\varepsilon}\left(a_{n}, \zeta\right)}\right) \leq H C^{2 \alpha} e^{-\alpha \varepsilon k\left(x_{n}, x_{n+1}\right)}
$$

or,

$$
e^{\varepsilon\left(\alpha k\left(x_{n}, x_{n+1}\right)-h\left(x_{n}, x_{n+1}\right)\right)} \leq H C^{1+2 \alpha},
$$

which in turn would imply that $\alpha k\left(x_{n}, x_{n+1}\right)-h\left(x_{n}, x_{n+1}\right)$ is bounded as $n \rightarrow+\infty$. However, $L_{n}:=\frac{1}{2} \log \left(x_{n+1} / x_{n}\right) \rightarrow+\infty$ as $n \rightarrow+\infty$, and,

$$
\alpha k\left(x_{n}, x_{n+1}\right)-h\left(x_{n}, x_{n+1} \geq 2 \alpha L_{n}-4-\pi \log L_{n}\right.
$$

from which we deduce that $\alpha k\left(x_{n}, x_{n+1}\right)-h\left(x_{n}, x_{n+1}\right)$ is not bounded as $n \rightarrow+\infty$. This contradiction means there cannot exist such a power quasisymmetry.

\section{Appendix A. Definitions of Uniformly PERfeCt}

Heinonen Hei01 has a general metric space definition for uniform perfectness (which appears as item A.1.(e) below) that is similar to, but different from, Pommerenke's definition. The following shows that the Pommerenke and Heinonen definitions are equivalent; this must be folklore, but we do not know a reference. Recall that a ring domain is a topological annulus, and a ring domain $D \subset \hat{\mathbb{C}}$ separates a set $E \subset \hat{\mathbb{C}}$ if and only if $D \subset \hat{\mathbb{C}} \backslash E$ and both components of $\hat{\mathbb{C}} \backslash D$ contain points of $E$.

A.1. Proposition. For any closed set $E \subset \hat{\mathbb{C}}$ with $\infty \in E$, the following are quantitatively equivalent:

(a) $E$ is $M$-uniformly perfect,

(b) bp $\leq M$ in $\Omega$ for each component $\Omega$ of $\hat{\mathbb{C}} \backslash E$,

(c) $\bmod (A) \leq M$ for any annulus $A \subset \hat{\mathbb{C}} \backslash E$ that separates $E$,

(d) $\bmod (D) \leq M$ for any ring domain $D \subset \hat{\mathbb{C}} \backslash E$ that separates $E$,

(e) $\hat{R} / \hat{r} \leq M$ for any $o \in E, \hat{R}>\hat{r}>0$ with $\mathrm{D}_{\chi}(o ; \hat{R}) \backslash \overline{\mathrm{D}}_{\chi}[o ; \hat{r}] \subset \hat{\mathbb{C}} \backslash E$ separating E.

The constant $M$ above will vary, but in each case it depends only on the other constants.

Proof. It is easy to check that (a) and (c) are equivalent. Beardon and Pommerenke [BP78] established the equivalence of (c) and (b), and Pommerenke [Pom79, Pom84] demonstrated that these are equivalent to (d) (along with several other variations).

That (e) is equivalent to the other conditions is surely well-known, yet does not seem to be explicitly mentioned in the literature, so we outline an explanation for this.

Suppose $o \in E, R>r>0$, and $D:=\mathrm{D}_{\chi}(o ; R) \backslash \overline{\mathrm{D}}_{\chi}[o ; r] \subset \hat{\mathbb{C}} \backslash E$ separates $E$. Then $D$ is a ring domain, so if (d) holds, then

$$
\log \frac{R}{r} \leq \log \frac{R \sqrt{4-r^{2}}}{r \sqrt{4-R^{2}}}=\bmod (D) \leq M .
$$


Finally, suppose (e) holds with a constant $M \geq 2$. To establish (a), we verify that (2.2) holds with the constant $64 M^{4}$. Let $A=\{r<|z-c|<R\} \subset \mathbb{C} \backslash E$ be an annulus with $c \in E \cap \mathbb{C}$; thus $A$ separates $E$. Assume $R \geq 2 r$. Roughly, we exhibit a chordal subannulus $\hat{A}$ a $A$ and use $\hat{R} / \hat{r} \leq M$ to bound $R / r$.

There are three cases depending on whether $|c| \geq R,|c| \leq r$, or $r<|c|<R$. Assume $|c| \geq R$. Here we verify that $R / r \leq 4 M$. Let

$$
a:=(|c|-r) \frac{c}{|c|}, b:=(|c|+R) \frac{c}{|c|}, \hat{r}:=\chi(a, c), \hat{R}:=\chi(b, c) .
$$

Since $|c|+R \leq 2|c|$ and $|c|-r \geq \frac{1}{2}|c|$,

$$
\frac{1+|b|^{2}}{1+|a|^{2}}=\frac{1+(|c|+R)^{2}}{1+(|c|-r)^{2}} \leq \frac{1+(2|c|)^{2}}{1+(|c| / 2)^{2}} \leq 16 .
$$

Thus if $\hat{r} \geq \hat{R}$, then $R / r \leq 4$; so we assume $\hat{r}<\hat{R}$. Then

$$
\hat{A}:=\{\hat{r}<\chi(z, c)<\hat{R}\} \text { a } A
$$

whence

$$
M \geq \frac{\hat{R}}{\hat{r}}=\frac{R}{r}\left(\frac{1+|a|^{2}}{1+|b|^{2}}\right)^{\frac{1}{2}}, \quad \text { so } \quad \frac{R}{r} \leq 4 M .
$$

Assume $|c| \leq r$. Here, eventually, we deduce that $R / r \leq 8 M^{2}$. Let

$$
a:=(|c|+r) \frac{c}{|c|}, b:=(|c|-R) \frac{c}{|c|}, \hat{r}:=\chi(a, 0), \hat{R}:=\chi(b, 0) .
$$

If $\hat{r} \geq \hat{R}$, then $\chi(b, \infty) \geq \chi(a, \infty)$, so again $R / r \leq 4$. Suppose $\hat{r}<\hat{R}$. Then

$$
\hat{A}:=\{\hat{r}<\chi(z, c)<\hat{R}\} \Subset \text { a } A, \text { whence } \frac{\hat{R}}{\hat{r}} \leq M .
$$

We claim that

$$
\sqrt{1+|a|^{2}} \geq \frac{\sqrt{2} M}{2 M-1} \quad \text { and } \quad \sqrt{1+|b|^{2}} \leq \sqrt{2} M
$$

therefore $M \geq \frac{\hat{R}}{\hat{r}}=\frac{|b|}{|a|}\left(\frac{1+|a|^{2}}{1+|b|^{2}}\right)^{\frac{1}{2}}$, so

$$
\frac{R / 2}{2 r} \leq \frac{R-|c|}{r+|c|}=\frac{|b|}{|a|} \leq\left(\frac{1+|b|^{2}}{1+|a|^{2}}\right)^{\frac{1}{2}} M \leq M(2 M-1)
$$

and hence $R / r \leq 8 M^{2}$.

To corroborate the above claim, we utilize chordal subannuli that have $|z|=1$ as a boundary circle. If $\sqrt{2} \leq \hat{r}=\chi(a, 0)$, then $|a| \geq 1$ so $\sqrt{1+|a|^{2}} \geq \sqrt{2} M /(2 M-1)$. Suppose $\hat{r}<\sqrt{2}$. Then

$$
\{\hat{r}<\chi(z, 0)<\sqrt{2}\} \text { a } A
$$


SO

$$
M \geq \frac{\sqrt{2}}{\hat{r}}=\frac{\sqrt{1+|a|^{2}}}{\sqrt{2}|a|} \leq \frac{1+|a|}{2|a|}
$$

and therefore

$$
\sqrt{1+|a|^{2}} \geq \frac{1+|a|}{\sqrt{2}} \geq \frac{\sqrt{2} M}{2 M-1} .
$$

If $\sqrt{2} \leq \hat{r}_{\infty}:=\chi(b, \infty)$, then $\sqrt{1+|b|^{2}} \leq \sqrt{2} \leq \sqrt{2} M$. Suppose $\hat{r}_{\infty}<\sqrt{2}$. Then

$$
\left\{\hat{r}_{\infty}<\chi(z, 0)<\sqrt{2}\right\} \text { a } A
$$

so $M \geq \frac{\sqrt{2}}{\hat{r}}=\frac{\sqrt{1+|b|^{2}}}{\sqrt{2}}$.

Thus when $|c| \leq r$ or $|c| \geq R, R / r \leq 8 M^{2}$. Suppose $r<|c|<R$, and $R \geq 4 r$. Then either $|c| \geq \sqrt{r R}$ or $|c| \leq \sqrt{r R}$. In either case, we can apply the previous arguments to the appropriate subannulus (either $\{r<|z-c|<\sqrt{R r}\}$ or $\{\sqrt{R r}<$ $|z-c|<R\})$ to conclude that in all cases $R / r \leq 64 M^{4}$.

\section{References}

[Aga68] Stephen Agard, Distortion theorems for quasiconformal mappings, Ann. Acad. Sci. Fenn. Ser. A I No. 413 (1968), 12. MR.0222288

[BB03] Zoltán M. Balogh and Stephen M. Buckley, Geometric characterizations of Gromov hyperbolicity, Invent. Math. 153 (2003), no. 2, 261-301, DOI 10.1007/s00222-003-02876. MR 1992014

[BP78] A. F. Beardon and Ch. Pommerenke, The Poincaré metric of plane domains, J. London Math. Soc. (2) 18 (1978), no. 3, 475-483, DOI 10.1112/jlms/s2-18.3.475. MR518232

[BHK01] Mario Bonk, Juha Heinonen, and Pekka Koskela, Uniformizing Gromov hyperbolic spaces, Astérisque 270 (2001), viii+99. MR1829896

[BS00] M. Bonk and O. Schramm, Embeddings of Gromov hyperbolic spaces, Geom. Funct. Anal. 10 (2000), no. 2, 266-306, DOI 10.1007/s000390050009. MR.1771428

[BH99] Martin R. Bridson and André Haefliger, Metric spaces of non-positive curvature, Grundlehren der Mathematischen Wissenschaften [Fundamental Principles of Mathematical Sciences], vol. 319, Springer-Verlag, Berlin, 1999. MR 1744486

[BH20] Stephen M. Buckley and David A. Herron, Quasihyperbolic geodesics are hyperbolic quasi-geodesics, J. Eur. Math. Soc. (JEMS) 22 (2020), no. 6, 1917-1970, DOI 10.4171/jems/959. MR 4092902

[BHX08] Stephen M. Buckley, David A. Herron, and Xiangdong Xie, Metric space inversions, quasihyperbolic distance, and uniform spaces, Indiana Univ. Math. J. 57 (2008), no. 2, 837-890. MR2414336

[BBI01] Dmitri Burago, Yuri Burago, and Sergei Ivanov, A course in metric geometry, Graduate Studies in Mathematics, vol. 33, American Mathematical Society, Providence, RI, 2001. MR.1835418

[Geh82] Frederick W. Gehring, Characteristic properties of quasidisks, Séminaire de Mathématiques Supérieures [Seminar on Higher Mathematics], vol. 84, Presses de l'Université de Montréal, Montreal, Que., 1982. MR674294

[GO79] F. W. Gehring and B. G. Osgood, Uniform domains and the quasihyperbolic metric, J. Analyse Math. 36 (1979), 50-74 (1980), DOI 10.1007/BF02798768. MR581801

[GP76] F. W. Gehring and B. P. Palka, Quasiconformally homogeneous domains, J. Analyse Math. 30 (1976), 172-199, DOI 10.1007/BF02786713. MR 437753

[Gol69] G. M. Goluzin, Geometric theory of functions of a complex variable, Translations of Mathematical Monographs, Vol. 26, American Mathematical Society, Providence, R.I., 1969. MR0247039

[Hei01] Juha Heinonen, Lectures on analysis on metric spaces, Universitext, Springer-Verlag, New York, 2001. MR:1800917 
[Hem79] Joachim A. Hempel, The Poincaré metric on the twice punctured plane and the theorems of Landau and Schottky, J. London Math. Soc. (2) 20 (1979), no. 3, 435-445, DOI 10.1112/jlms/s2-20.3.435. MR.561135

[Her84] David Alan Herron, Conformally Invariant Metrics and the Geometry of Uniform Domains (Distance, Harnack), ProQuest LLC, Ann Arbor, MI, 1984. Thesis (Ph.D.)University of Michigan. MR2633627

[Her10] David A. Herron, Geometry and topology of intrinsic distances, J. Anal. 18 (2010), 197-231. MR2850242

[Her21a] D. A. Herron, Quasihyperbolic metric universal covers, Rev. Mat. Iberoamericana, online-first 2020-11-25 DOI: 10.4171/rmi/1226, 30 pages.

[Her21b] D. A. Herron, Uniform domains and hyperbolic distance, J. Analyse Math. (2021), DOI:10.1007/s11854-021-0160-9.

[HLM89] David A. Herron, Xiang Yang Liu, and David Minda, Ring domains with separating circles or separating annuli, J. Analyse Math. 53 (1989), 233-252, DOI 10.1007/BF02793416. MR,1014988

[HRS20] David A. Herron, Abigail Richard, and Marie A. Snipes, Chordal Hausdorff convergence and quasihyperbolic distance, Anal. Geom. Metr. Spaces 8 (2020), no. 1, 36-67, DOI 10.1515/agms-2020-0104. MR4118580

[Jen81] James A. Jenkins, On explicit bounds in Landau's theorem. II, Canadian J. Math. 33 (1981), no. 3, 559-562, DOI 10.4153/CJM-1981-045-1. MR627642

[Jon80] Peter W. Jones, Extension theorems for BMO, Indiana Univ. Math. J. 29 (1980), no. 1, 41-66, DOI 10.1512/iumj.1980.29.29005. MR554817

[Jon81] Peter W. Jones, Quasiconformal mappings and extendability of functions in Sobolev spaces, Acta Math. 147 (1981), no. 1-2, 71-88, DOI 10.1007/BF02392869. MR631089

[LVV59] Olli Lehto, K. I. Virtanen, and Jussi Väisälä, Contributions to the distortion theory of quasiconformal mappings, Ann. Acad. Sci. Fenn. Ser. A I No. 273 (1959), 14. MR.0122990

[MO86] Gaven J. Martin and Brad G. Osgood, The quasihyperbolic metric and associated estimates on the hyperbolic metric, J. Analyse Math. 47 (1986), 37-53, DOI 10.1007/BF02792531. MR874043

[MS79] O. Martio and J. Sarvas, Injectivity theorems in plane and space, Ann. Acad. Sci. Fenn. Ser. A I Math. 4 (1979), no. 2, 383-401, DOI 10.5186/aasfm.1978-79.0413. MR.565886

[Min87] David Minda, Inequalities for the hyperbolic metric and applications to geometric function theory, Complex analysis, I (College Park, Md., 1985), Lecture Notes in Math., vol. 1275, Springer, Berlin, 1987, pp. 235-252, DOI 10.1007/BFb0078356. MR922304

[Pom79] Ch. Pommerenke, Uniformly perfect sets and the Poincaré metric, Arch. Math. (Basel) 32 (1979), no. 2, 192-199, DOI 10.1007/BF01238490. MR534933

[Pom84] Ch. Pommerenke, On uniformly perfect sets and Fuchsian groups, Analysis 4 (1984), no. 3-4, 299-321, DOI 10.1524/anly.1984.4.34.299. MR780609

[SV01] A. Yu. Solynin and M. Vuorinen, Estimates for the hyperbolic metric of the punctured plane and applications, Israel J. Math. 124 (2001), 29-60, DOI 10.1007/BF02772606. MR.1856503

[Sug01] Toshiyuki Sugawa, Uniformly perfect sets-their analytical and geometrical aspects (Japanese), Sūgaku 53 (2001), no. 4, 387-402. MR1869018

[SV05] Toshiyuki Sugawa and Matti Vuorinen, Some inequalities for the Poincaré metric of plane domains, Math. Z. 250 (2005), no. 4, 885-906, DOI 10.1007/s00209-005-0782-0. MR2180380

[Tsu75] M. Tsuji, Potential theory in modern function theory, Chelsea Publishing Co., New York, 1975. Reprinting of the 1959 original. MR0414898

[TV80] P. Tukia and J. Väisälä, Quasisymmetric embeddings of metric spaces, Ann. Acad. Sci. Fenn. Ser. A I Math. 5 (1980), no. 1, 97-114, DOI 10.5186/aasfm.1980.0531. MR595180

[Väi94] Jussi Väisälä, Exhaustions of John domains, Ann. Acad. Sci. Fenn. Ser. A I Math. 19 (1994), no. 1, 47-57. MR 1246886

[Väi05] Jussi Väisälä, Gromov hyperbolic spaces, Expo. Math. 23 (2005), no. 3, 187-231, DOI 10.1016/j.exmath.2005.01.010. MR.2164775 
Department of Mathematical Sciences, University of Cincinnati, Cincinnati, Ohio 45221-0025

Email address: David.Herron@UC.edu

Department of Mathematical Sciences, University of Cincinnati, Cincinnati, Ohio 45221-0025

Email address: jlindquistmath@gmail.com 